\title{
The off-shell 4D/5D connection
}

\author{
Nabamita Banerjee, ${ }^{a}$ Bernard de Wit ${ }^{a, b}$ and Stefanos Katmadas ${ }^{c}$ \\ ${ }^{a}$ Institute for Theoretical Physics, Utrecht University, \\ Leuvenlaan 4, 3584 CE Utrecht, The Netherlands \\ ${ }^{b}$ Nikhef Theory Group, \\ Science Park 105, 1098 XG Amsterdam, The Netherlands \\ ${ }^{c}$ Centre de Physique Théorique, Ecole Polytechnique, CNRS, \\ 91128 Palaiseau cedex, France \\ E-mail: N.Banerjee@uu.nl, B.deWit@uu.nl, \\ katmadas@cpht.polytechnique.fr
}

Abstract: A systematic off-shell reduction scheme from five to four space-time dimensions is presented for supergravity theories with eight supercharges. It is applicable to theories with higher-derivative couplings and it is used to address a number of open questions regarding BPS black holes in five dimensions. Under this reduction the $5 D$ Weyl multiplet becomes reducible and decomposes into the $4 D$ Weyl multiplet and an extra Kaluza-Klein vector multiplet. The emergence of the pseudoscalar field of the latter multiplet and the emergence of the $4 D$ R-symmetry group are subtle features of the reduction. The reduction scheme enables to determine how a $5 D$ supersymmetric Lagrangian with higher-derivative couplings decomposes upon dimensional reduction into a variety of independent $4 D$ supersymmetric invariants, without the need for imposing field equations. In this way we establish, for example, the existence of a new $\mathrm{N}=2$ supersymmetric invariant that involves the square of the Ricci tensor. Finally we resolve the questions associated with the $5 D$ Chern-Simons terms for spinning BPS black holes and their relation to the corresponding $4 D$ black holes.

KEYwords: Gauge Symmetry, Black Holes, Supergravity Models

ARXIV EPRINT: 1112.5371 


\section{Contents}

1 Introduction 1

2 Off-shell dimensional reduction; the Weyl multiplet $\quad 3$

3 Off-shell dimensional reduction; matter multiplets 13

$4 \quad$ Five and four-dimensional fields and invariant Lagrangians $\quad 16$

5 The 4D/5D connection and the BPS spinning black hole 23

$\begin{array}{llr}6 & \text { Concluding remarks } & 29\end{array}$

$\begin{array}{ll}\text { A Relations between 5D and 4D Riemann curvatures } & 30\end{array}$

B Conversion of $5 D$ symplectic Majorana spinors into $4 D$ chiral spinors $\quad 32$

C Supersymmetry transformations in four dimension 33

\section{Introduction}

Dimensional reduction plays an important role in the study of many aspects of supergravity and string theory. Usually the reduction is performed in the context of supersymmetric on-shell field configurations. For theories with a large number of supercharges this is unavoidable, as off-shell representations are usually not available. For theories based on off-shell representations there has been little effort so far to define a suitable dimensional reduction scheme, because the extra auxiliary fields contained in the off-shell configuration can be ignored upon solving their corresponding (algebraic) field equations. However, in the presence of higher-derivative couplings, these field equations are no longer algebraic. In their on-shell form these couplings will therefore take the form of an iterative expansion in increasing powers of space-time derivatives, which obscures the structure of the underlying off-shell invariants.

Dimensional reduction of off-shell configurations is based on a corresponding reduction of the off-shell supersymmetry algebra. It can therefore be performed systematically on separate multiplets. To accomplish this one maps a supermultiplet in higher dimension on a corresponding, not necessarily irreducible, supermultiplet in lower dimension, possibly in a certain supergravity background. When considering the supersymmetry algebra in the context of a lower-dimensional space-time, the dimension of the automorphism group 
of the algebra (the R-symmetry group) usually increases, and this has to be taken into account when casting the resulting supermultiplet in its standard form. The fact that irreducible multiplets in higher dimension can become reducible in lower dimensions, further complicates the reduction procedure.

In this paper we study the off-shell dimensional reduction of $5 D N=1$ superconformal multiplets to the corresponding $N=2$ superconformal multiplets in four dimensions. Onshell dimensional reduction of these theories has been considered in the past for a variety of reasons, but mainly in the context of actions that are at most quadratic in space-time derivatives. For some earlier work we refer to [1-4]. We choose the superconformal setting because this enables us to work in the context of the superconformal multiplet calculus, which has been developed in both four and five dimensions. ${ }^{1}$ It may seem that conformal invariance will be an impediment to dimensional reduction, because conformal symmetry depends strongly on the actual space-time dimension. However, it turns out that this is not problematic at all, because the conformal transformations are formulated in a way that is independent on the four- or five-dimensional space-time manifold (which is subject to general diffeomorphisms) whereas the scale transformations and conformal boosts are defined in the tangent space. We will not elaborate on this aspect in further detail as it will be rather explicit in the construction that we are about to present. The construction is somewhat facilitated by the fact that the spinor dimension is the same in five and in four dimensions: in four dimensions we are dealing with a doublet of four-component independent Majorana spinors, and in five dimensions we have a four-component spinor, which can be treated either as a Dirac spinor or as a symplectic Majorana spinor. Both these spinors share a common $\mathrm{SU}(2)$ factor in the R-symmetry group. We will exhibit in detail how the additional U(1) factor will emerge in four dimensions. Here we recall that in conformal supergravity, R-symmetry is realized as a local symmetry.

The whole reduction scheme is subtle, especially in view of the fact that the $5 D$ Weyl multiplet decomposes into a $4 D$ Weyl multiplet and an additional vector multiplet. In spite of this, both in five and in four dimensions, the matter multiplets are defined in a superconformal background consisting only of the $5 D$ or the $4 D$ Weyl multiplet fields, respectively. To fully establish this fact requires to also consider the transformation rules beyond the linearized approximation. As an aside we mention that a corresponding reduction from four to three dimensions will involve a further extension of the R-symmetry group. Namely, $\mathrm{SU}(2) \times \mathrm{U}(1)$ will then be elevated to the group $\mathrm{SU}(2) \times \mathrm{SU}(2)$.

The central result of this paper will be to express the $5 D$ off-shell fields in terms of the $4 D$ ones. We then verify that the $5 D$ supersymmetric actions with terms quadratic in derivatives will yield the $4 D$ ones, at least for the bosonic fields. Subsequently we consider the $5 D$ action with terms quartic in derivatives [6] and evaluate a number of characteris-

\footnotetext{
${ }^{1}$ In [5], off-shell dimensional reduction in $6 D$ was used to determine the superconformal transformations in $5 D$.
} 
tic terms in the reduction to four dimensions to properly identify the $4 D$ invariants that emerge. As it turns out the result decomposes into at least three different invariants with higher derivatives. One of them is the invariant based on a chiral superspace integral (the so-called 'F-term') [7, 8], another one the invariant based on a full superspace integral (the so-called 'D-term') [9], and finally a (not necessarily irreducible) invariant emerges that involves the square of the Ricci tensor, which could in principle appear as an $N=2$ supercovariantization of the Gauss-Bonnet term.

These results enable us to address a number of issues that arose from previous work on spinning BPS black holes in five dimensions and their possible relation to four-dimensional black holes [10-13]. In this discussion the invariants with higher-derivative couplings play a major role. Using a simplified model we find further support for the results presented in [13] and explain the subtle issues for spinning black holes associated with the mixed gauge-gravitational Chern-Simons term.

This paper is organized as follows. Section 2 presents the off-shell reduction to four space-time dimensions of the five-dimensional Weyl multiplet, and section 3 presents the corresponding results for the vector multiplet and the hypermultiplet. Section 4 takes into account the conversion of $5 D$ symplectic Majorana spinors to the $4 D$ chiral spinor to obtain the explicit relations between $4 D$ and $5 D$ fields. Subsequently these results are applied to supersymmetric actions, leading to the precise decomposition of the $5 D$ Lagrangians into $4 D$ supersymmetric Lagrangians. Section 5 addresses the situation regarding BPS black holes, where differences were noted in the attractor equations for the electric charges in the presence of higher-derivative couplings. There are three appendices. Appendix A discusses the relation between $5 D$ and $4 D$ Riemann curvatures, the conversion of $5 D$ spinors to $4 D$ chiral spinors is presented in appendix $\mathrm{B}$, and some $4 D$ supersymmetry transformations are collected in appendix $\mathrm{C}$.

\section{Off-shell dimensional reduction; the Weyl multiplet}

Starting from the superconformal transformations for $5 D$ supermultiplets we will reduce the space-time dimension to $4 D$ and reinterpret the results in terms of the known superconformal transformations in $4 D$ dimensions. The first multiplet to consider is the Weyl multiplet, because it acts as a background for other supermultiplets, such as the vector multiplet and the hypermultiplet. A second reason why the Weyl multiplet deserves priority, is that it becomes reducible upon the reduction, unlike the other (matter) supermultiplets. The Weyl multiplet in $D=5$ comprises $32+32$ bosonic and fermionic degrees of freedom, which, in the reduction to $D=4$ dimensions decomposes into the Weyl multiplet comprising $24+24$ degrees of freedom, and a vector multiplet comprising $8+8$ degrees of freedom. As we shall see, this decomposition takes a subtle form off-shell.

The independent fields of the Weyl multiplet of five-dimensional $N=1$ conformal supergravity consist of the fünfbein $e_{M}{ }^{A}$, the gravitino fields $\psi_{M}{ }^{i}$, the dilatational gauge 
field $b_{M}$, the R-symmetry gauge fields $V_{M i}{ }^{j}$ (which is an anti-hermitean, traceless matrix in the $\mathrm{SU}(2)$ indices $i, j$ ) and a tensor field $T_{A B}$, a scalar field $D$ and a spinor field $\chi^{i}$. All spinor fields are symplectic Majorana spinors. Our conventions are as in [13] apart from the supersymmetry parameters $\epsilon^{i}$ which have been rescaled by a factor of two to bring the normalization of the $5 D$ supersymmetry algebra in line with the $4 D$ algebra. The three gauge fields $\omega_{M}{ }^{A B}, f_{M}{ }^{A}$ and $\phi_{M}{ }^{i}$, associated with local Lorentz transformations, conformal boosts and S-supersymmetry, respectively, are not independent as will be discussed later. The infinitesimal Q, S and K transformations of the independent fields, parametrized by spinors $\epsilon^{i}$ and $\eta^{i}$ and a vector $\Lambda_{\mathrm{K}}{ }^{A}$, respectively, are as follows, ${ }^{2}$

$$
\begin{aligned}
\delta e_{M}{ }^{A}= & \bar{\epsilon}_{i} \gamma^{A} \psi_{M}{ }^{i} \\
\delta \psi_{M}{ }^{i}= & 2 \mathcal{D}_{M} \epsilon^{i}+\frac{1}{2} \mathrm{i} T_{A B}\left(3 \gamma^{A B} \gamma_{M}-\gamma_{M} \gamma^{A B}\right) \epsilon^{i}-\mathrm{i} \gamma_{M} \eta^{i} \\
\delta V_{M i}{ }^{j}= & 6 \mathrm{i} \bar{\epsilon}_{i} \phi_{M}{ }^{j}-16 \bar{\epsilon}_{i} \gamma_{M} \chi^{j}-3 \mathrm{i} \bar{\eta}_{i} \psi_{M}{ }^{j}+\delta^{i}{ }_{j}\left[-3 \mathrm{i} \bar{\epsilon}_{k} \phi_{M}{ }^{k}+8 \bar{\epsilon}_{k} \gamma_{M} \chi^{k}+\frac{3}{2} \mathrm{i} \bar{\eta}_{k} \psi_{M}{ }^{k}\right] \\
\delta b_{M}= & \mathrm{i} \bar{\epsilon}_{i} \phi_{M}{ }^{i}-4 \bar{\epsilon}_{i} \gamma_{M} \chi^{i}+\frac{1}{2} \mathrm{i} \bar{\eta}_{i} \psi_{M}{ }^{i}+2 \Lambda_{K}{ }^{A} e_{M A} \\
\delta T_{A B}= & \frac{4}{3} \mathrm{i} \bar{\epsilon}_{i} \gamma_{A B} \chi^{i}-\frac{1}{4} \mathrm{i} \bar{\epsilon}_{i} R_{A B}{ }^{i}(Q) \\
\delta \chi^{i}= & \frac{1}{2} \epsilon^{i} D+\frac{1}{64} R_{M N j}{ }^{i}(V) \gamma^{M N} \epsilon^{j}+\frac{3}{64} \mathrm{i}\left(3 \gamma^{A B} \not D+\not D \gamma^{A B}\right) T_{A B} \epsilon^{i} \\
& -\frac{3}{16} T_{A B} T_{C D} \gamma^{A B C D} \epsilon^{i}+\frac{3}{16} T_{A B} \gamma^{A B} \eta^{i} \\
\delta D= & 2 \bar{\epsilon}_{i} \not D \chi^{i}-2 \mathrm{i} \bar{\epsilon}_{i} T_{A B} \gamma^{A B} \chi^{i}-\mathrm{i} \bar{\eta}_{i} \chi^{i}
\end{aligned}
$$

Under local scale transformations the various fields and transformation parameters transform as indicated in table 1 . The derivatives $\mathcal{D}_{M}$ are covariant with respect to all the bosonic gauge symmetries with the exception of the conformal boosts. In particular we note

$$
\mathcal{D}_{M} \epsilon^{i}=\left(\partial_{M}-\frac{1}{4} \omega_{M}{ }^{C D} \gamma_{C D}+\frac{1}{2} b_{M}\right) \epsilon^{i}+\frac{1}{2} V_{M j}{ }^{i} \epsilon^{j},
$$

where the gauge fields transform under their respective gauge transformations according to $\delta \omega_{M}{ }^{A B}=\mathcal{D}_{M} \lambda^{A B}, \delta b_{M}=\mathcal{D}_{M} \Lambda_{D}$ and $\delta V_{M i}{ }^{j}=\mathcal{D}_{M} \Lambda_{i}{ }^{j}$, with $\left(\Lambda_{i}{ }^{j}\right)^{*} \equiv \Lambda^{i}{ }_{j}=-\Lambda_{j}{ }^{i}$. The derivatives $D_{M}$ are covariant with respect to all the superconformal symmetries.

The above supersymmetry variations and also the conventional constraints that we have to deal with in due time, depend on a number of supercovariant curvature tensors, which take the following form,

$$
\begin{aligned}
R(P)_{M N}{ }^{A} & =2 \mathcal{D}_{[M} e_{N]}^{A}-\frac{1}{2} \bar{\psi}_{M i} \gamma^{A} \psi_{N}{ }^{i}, \\
R(M)_{M N}{ }^{A B} & =2 \partial_{[M} \omega_{N]}^{A B}-2 \omega_{[M}^{A C} \omega_{N] C}^{B}-8 e_{\left[M^{[A} f_{N]}{ }^{B]}+\mathrm{i} \bar{\psi}_{[M i} \gamma^{A B} \phi_{N]}^{i}\right.}^{i}
\end{aligned}
$$

\footnotetext{
${ }^{2}$ In five dimensions we consistently use world indices $M, N, \ldots$ and tangent space indices $A, B, \ldots$ For fields that do not carry such indices the distinction between $5 D$ and $4 D$ fields may not always be manifest, but it will be specified in the text whenever necessary.
} 


\begin{tabular}{|c|ccccccc|ccc|cc|}
\hline & \multicolumn{10}{|c|}{ Weyl multiplet } & \multicolumn{2}{|c|}{ parameters } \\
\hline field & $e_{M}{ }^{A}$ & $\psi_{M}{ }^{i}$ & $b_{M}$ & $V_{M i}{ }^{j}$ & $T_{A B}$ & $\chi^{i}$ & $D$ & $\omega_{M}{ }^{A B}$ & $f_{M}{ }^{A}$ & $\phi_{M}{ }^{i}$ & $\epsilon^{i}$ & $\eta^{i}$ \\
\hline$w$ & -1 & $-\frac{1}{2}$ & 0 & 0 & 1 & $\frac{3}{2}$ & 2 & 0 & 1 & $\frac{1}{2}$ & $-\frac{1}{2}$ & $\frac{1}{2}$ \\
\hline
\end{tabular}

Table 1. Weyl weights $w$ of the Weyl multiplet component fields and the supersymmetry transformation parameters.

$$
\begin{aligned}
& -\frac{1}{4} \mathrm{i} T^{C D} \bar{\psi}_{[M i}\left(6 \gamma^{[A} \gamma_{C D} \gamma^{B]}-\gamma^{A B} \gamma_{C D}-\gamma_{C D} \gamma^{A B}\right) \psi_{N]}^{i} \\
& -\frac{1}{2} \bar{\psi}_{[M i}\left(\gamma_{N]} R^{A B i}(Q)+2 \gamma^{[A} R_{N]}^{B] i}(Q)\right)+8 e_{\left[M^{[A}\right.} \bar{\psi}_{N] i} \gamma^{B]} \chi^{i}, \\
& R(D)_{M N}=2 \partial_{[M} b_{N]}-4 f_{[M}^{A} e_{N] A}-\mathrm{i} \bar{\psi}_{[M i} \phi_{N]}^{i}+4 \bar{\psi}_{[M i} \gamma_{N]} \chi^{i} . \\
& R(V)_{M N i}{ }^{j}=2 \partial_{[M} V_{N] i}{ }^{j}-V_{[M i}{ }^{k} V_{N] k}{ }^{j} \\
& -6 \mathrm{i} \bar{\psi}_{[M i} \phi_{N]}^{j}+16 \bar{\psi}_{[M i} \gamma_{N]} \chi^{j}+\delta_{i}{ }^{j}\left[3 \mathrm{i} \bar{\psi}_{[M k} \phi_{N]}{ }^{k}-8 \bar{\psi}_{[M k} \gamma_{N]} \chi^{k}\right], \\
& R(Q)_{M N}^{i}=2 \mathcal{D}_{[M} \psi_{N]}^{i}-2 \mathrm{i} \gamma_{[M} \phi_{N]}^{i}+\frac{1}{2} \mathrm{i} T_{A B}\left(3 \gamma^{A B} \gamma_{[M}-\gamma_{[M} \gamma^{A B}\right) \psi_{N]}^{i}
\end{aligned}
$$

The curvature tensor $R_{M N}{ }^{A}(K)$ associated with the conformal boosts has not been defined and is not needed henceforth. The curvature tensor $R_{M N}{ }^{i}(S)$ will be discussed shortly.

The conventional constraints are as follows,

$$
\begin{aligned}
R(P)_{M N}{ }^{A} & =0, \\
\gamma^{M} R(Q)_{M N^{i}} & =0, \\
e_{A}{ }^{M} R(M)_{M N}{ }^{A B} & =0 .
\end{aligned}
$$

These conditions determine the gauge fields $\omega_{M}{ }^{A B}, f_{M}{ }^{A}$ and $\phi_{M}{ }^{i}$. The conventional constraints lead to additional constraints on the curvatures when combined with the Bianchi identities. In this way one derives $R(M)_{[A B C] D}=0=R(D)_{A B}$ and the pair-exchange property $R(M)_{A B C D}=R(M)_{C D A B}$ from the first and the third constraint. The second constraint, which implies also that $\left.\gamma_{[M N} R(Q)_{P Q}\right]^{i}=0$, determines the curvature $R(S)_{M N}{ }^{i}$, which we refrained from defining previously. It turns out to be proportional to $R(Q)_{M N}{ }^{i}$ and derivatives thereof,

$$
\begin{aligned}
& R(S)_{M N}^{i}=-\mathrm{i} \not D R(Q)_{M N}{ }^{i}-\mathrm{i} \gamma_{[M} D^{P} R(Q)_{N] P}{ }^{i}-4 \gamma_{M N} T^{P Q} R(Q)_{P Q}{ }^{i} \\
& +18 T^{P Q} \gamma_{Q} \gamma_{[M} R(Q)_{N] P}{ }^{i}-5 T^{P Q} \gamma_{P Q} R(Q)_{M N}{ }^{i}-12 T^{P}{ }_{[M} R(Q)_{N] P}{ }^{i} .
\end{aligned}
$$

The reduction to four space-time dimensions is effected by first carrying out the standard Kaluza-Klein decompositions on the various fields, to guarantee that the resulting $4 D$ fields will transform consistently under four-dimensional diffeomorphisms. The space-time coordinates are decomposed into $x^{M} \rightarrow\left(x^{\mu}, x^{5}\right)$, where $x^{5}$ denotes the fifth coordinate that will be suppressed in the reduction. Subsequently the vielbein field and the dilatational 
gauge field are then written in special form, by means of an appropriate local Lorentz transformation and a conformal boost, respectively. In obvious notation,

$$
e_{M}^{A}=\left(\begin{array}{cc}
e_{\mu}{ }^{a} & B_{\mu} \phi^{-1} \\
0 & \phi^{-1}
\end{array}\right), \quad e_{A}^{M}=\left(\begin{array}{cc}
e_{a}{ }^{\mu}-e_{a}{ }^{\nu} B_{\nu} \\
0 & \phi
\end{array}\right), \quad b_{M}=\left(\begin{array}{c}
b_{\mu} \\
0
\end{array}\right) .
$$

On the right-hand side of these decompositions, we exclusively used four-dimensional notation, with world and tangent-space indices, $\mu, \nu, \ldots$ and $a, b, \ldots$, taking four values. Observe that the scaling weights for $e_{M}{ }^{A}$ and $e_{\mu}{ }^{a}$ are equal to $w=-1$, while for $\phi$ we have $w=1$. The fields $b_{M}$ and $b_{\mu}$ have weight $w=0$. The above formulae suffice to express the $5 D$ Riemann curvature tensor in terms of the $4 D$ Riemann tensor and the fields $\phi$ and $B_{\mu}$. The corresponding equations are collected in appendix $\mathrm{A}$ and will be needed later on.

We now turn to the supersymmetry transformations. Since we have imposed gauge choices on the vielbein field and the dilatational gauge field, one has to include compensating Lorentz and special conformal transformations when deriving the $4 D$ Q-supersymmetry transformations to ensure that the gauge conditions are preserved. Only the parameter of the Lorentz transformation is relevant, and it is given by,

$$
\varepsilon^{a 5}=-\varepsilon^{5 a}=-\phi \bar{\epsilon}_{i} \gamma^{a} \psi^{i},
$$

where we assumed the standard Kaluza-Klein decomposition on the gravitino fields,

$$
\psi_{M^{i}}=\left(\begin{array}{c}
\psi_{\mu}^{i}+B_{\mu} \psi^{i} \\
\psi^{i}
\end{array}\right) \text {, }
$$

which ensures that $\psi_{\mu}{ }^{i}$ on the right-hand side transforms as a $4 D$ vector. Upon including this extra term, one can write down the Q- and S-supersymmetry transformations on the $4 D$ fields defined above. As a result of this, the $4 D$ and $5 D$ supersymmetry transformation will be different. For instance, the supersymmetry transformations of the $4 D$ fields $e_{\mu}{ }^{a}, \phi$ and $B_{\mu}$ read,

$$
\begin{aligned}
\delta e_{\mu}{ }^{a} & =\bar{\epsilon}_{i} \gamma^{a} \psi_{\mu}{ }^{i}, \\
\delta \phi & =-\phi^{2} \bar{\epsilon}_{i} \gamma^{5} \psi^{i}, \\
\delta B_{\mu} & =\phi^{2} \bar{\epsilon}_{i} \gamma_{\mu} \psi^{i}+\phi \bar{\epsilon}_{i} \gamma^{5} \psi_{\mu}{ }^{i},
\end{aligned}
$$

where the first term in $\delta B_{\mu}$ originates from the compensating transformation (2.7). Consequently the supercovariant field strength of $B_{\mu}$ contains a term that is not contained in the supercovariant five-dimensional curvature $R(P)_{M N}{ }^{A}$. Therefore the $5 D$ spin-connection components are not supercovariant with respect to $4 D$ supersymmetry, as is shown below,

$$
\omega_{M}^{a b}=\left(\begin{array}{c}
\omega_{\mu}^{a b} \\
0
\end{array}\right)+\frac{1}{2} \phi^{-2} \hat{F}(B)^{a b}\left(\begin{array}{c}
B_{\mu} \\
1
\end{array}\right),
$$




$$
\omega_{M}^{a 5}=-\frac{1}{2}\left(\begin{array}{c}
\phi^{-1} \hat{F}(B)_{\mu}^{a}+\phi \bar{\psi}_{\mu i} \gamma^{a} \psi^{i} \\
0
\end{array}\right)-\phi^{-2} D^{a} \phi\left(\begin{array}{c}
B_{\mu} \\
1
\end{array}\right) .
$$

Here we introduced the supercovariant field strength and derivative (with respect to $4 D$ supersymmetry),

$$
\begin{aligned}
\hat{F}(B)_{\mu \nu} & =2 \partial_{[\mu} B_{\nu]}-\phi^{2} \bar{\psi}_{[\mu i} \gamma_{\nu]} \psi^{i}-\frac{1}{2} \phi \bar{\psi}_{\mu i} \psi_{\nu}{ }^{i}, \\
D_{\mu} \phi & =\left(\partial_{\mu}-b_{\mu}\right) \phi+\frac{1}{2} \phi^{2} \bar{\psi}_{\mu i} \gamma_{5} \psi^{i} .
\end{aligned}
$$

Subsequently we write down corresponding Kaluza-Klein decompositions for some of the other fields of the Weyl multiplet, which do not require special gauge choices,

$$
V_{M i}{ }^{j}=\left(\begin{array}{c}
V_{\mu i}{ }^{j}+B_{\mu} V_{i}^{j} \\
V_{i}^{j}
\end{array}\right), \quad \phi_{M}{ }^{i}=\left(\begin{array}{c}
\phi_{\mu}{ }^{i}+B_{\mu} \phi^{i} \\
\phi^{i}
\end{array}\right) \quad T_{A B}=\left(\begin{array}{c}
T_{a b} \\
T_{a 5} \equiv-\frac{1}{6} A_{a}
\end{array}\right) .
$$

For the fermions there is yet no need to introduce new notation, because the spinors have an equal number of components in five and four space-time dimensions. Eventually we will convert to standard four-dimensional chiral spinors.

Hence we are now ready to consider the supersymmetry transformations of the spinor fields originating from the $5 D$ gravitino fields. Up to possible higher-order spinor terms, one derives from (2.1),

$$
\begin{aligned}
\delta \psi^{i}= & -\frac{1}{2} \phi^{-2}\left[\hat{F}(B)_{a b}-\mathrm{i} \gamma_{5} \phi\left(3 T_{a b}-\frac{1}{4} \mathrm{i} \phi^{-1} \hat{F}(B)_{a b} \gamma_{5}\right)\right] \gamma^{a b} \epsilon^{i} \\
& +\phi^{-2}\left[\not D \phi \gamma^{5}-\mathrm{i} A \phi\right] \epsilon^{i}-V^{i}{ }_{j} \epsilon^{j} \\
& -\mathrm{i} \gamma_{5} \phi^{-1}\left[\eta^{i}+\frac{1}{3} A \gamma_{5} \epsilon^{i}+\frac{1}{8} \mathrm{i} \gamma_{5} \phi^{-1}\left(F(B)_{a b}-4 \mathrm{i} \phi T_{a b} \gamma_{5}\right) \gamma^{a b} \epsilon^{i}\right], \\
\delta \psi_{\mu}{ }^{i}= & 2\left(\partial_{\mu}-\frac{1}{4} \omega_{\mu}{ }^{a b} \gamma_{a b}+\frac{1}{2} b_{\mu}+\frac{1}{2} \mathrm{i} e_{\mu}{ }^{a} A_{a} \gamma_{5}\right) \epsilon^{i}+V_{\mu j}{ }^{i} \epsilon^{j} \\
& +\frac{1}{2} \mathrm{i}\left[3 T_{a b}-\frac{1}{4} \mathrm{i} \phi^{-1} \hat{F}(B)_{a b} \gamma_{5}\right] \gamma_{a b} \gamma_{\mu} \epsilon^{i} \\
& -\mathrm{i} \gamma_{\mu}\left[\eta^{i}+\frac{1}{3} A A \gamma_{5} \epsilon^{i}+\frac{1}{8} \mathrm{i} \gamma_{5} \phi^{-1}\left(\hat{F}(B)_{a b}-4 \mathrm{i} \phi T_{a b} \gamma_{5}\right) \gamma^{a b} \epsilon^{i}\right] .
\end{aligned}
$$

Although this result is not yet complete, it already exhibits some of the systematic features that will turn out to be universal. Therefore let us have a brief perusal of these initial results.

The fields whose transformations we have determined will belong to two $4 D$ supermultiplets, namely the Weyl and the Kaluza-Klein vector multiplet. Clearly, the fields $e_{\mu}{ }^{a}$ and $\psi_{\mu}{ }^{i}$ belong to the Weyl multiplet, whereas $\phi, B_{\mu}$ and $\psi^{i}$ belong to the vector multiplet. Their transformations shown in (2.9) and (2.13) have many features in common with the 
standard $4 D$ transformations of a Weyl and a vector multiplet. An obvious puzzle is the fact that we have identified only one real scalar, whereas the $D=4$ vector multiplet contains a complex scalar. Furthermore, we note that the field $A_{a}$ seems to play the role of a $\mathrm{U}(1)$ gauge field, because it appears to covariantize the derivatives on $\phi$ and $\epsilon^{i}$ in (2.13), in spite of the fact that it is actually an auxiliary field in $D=5$. As we shall see in due course, the resolution of these two problems is related.

Another observation is that a particular linear combination of the $5 D$ tensor components $T_{a b}$ and the supercovariant field strength $\hat{F}(B)_{a b}$ appears in the transformations (2.13) in precisely the same form as the $4 D$ auxiliary tensor $T_{a b}$, suggesting that the latter is not just proportional to the corresponding $5 D$ tensor field components. The same combination will also appear in other transformation rules, as we shall see in, for instance, section 3. Finally, S-supersymmetry transformations are accompanied by another universal combination of $T_{a b}$ and $\hat{F}(B)_{a b}$. Obviously such a field-dependent component in the S-supersymmetry transformation can be dropped provided that it appears universally for all other fields, as it can be absorbed into $\eta^{i}$.

As it turns out this pattern becomes more complicated when including terms of higher order in the fermions. Apart from new contributions to the expressions noted above, it turns out that also R-symmetry will appear on the right-hand side with parameters that involve the spinors $\psi^{i}$. Again this R-symmetry transformation acts universally on all the fields. Hence the conclusion is that the $5 D$ supersymmetries decompose under the reduction into the $4 D$ supersymmetries up to $4 D$ field-dependents S-supersymmetries and $\mathrm{SU}(2)$ R-symmetries. This property explains why only a careful analysis can reveal how the offshell supermultiplets reduce to lower dimension, as precise knowledge of this decomposition is required before one can reliably extract the $4 D$ transformations. These transformations will then subsequently identify the $4 D$ fields in terms of the $5 D$ ones (up to straightforward calibrations). After verifying that the decomposition is universally realized these extra symmetries with field-dependent coefficients can be dropped.

However, there is yet another surprise, as we will discover the presence of a chiral $\mathrm{U}(1)$ transformation in the supersymmetry variations with a universal coefficient. Since chiral $\mathrm{U}(1)$ does not constitute a symmetry of the $5 D$ theory, the contribution from this transformation cannot be dropped and should be kept until the end. We will discuss its fate in due time. Obviously these transformations will play a crucial role in extending the R-symmetry to $\mathrm{SU}(2) \times \mathrm{U}(1)$.

Summarizing, we intend to first establish that the dimensional reduction of the $5 D$ supersymmetry variations, according to the procedure sketched above, takes the form of the $4 D$ supersymmetry variations combined with a field-dependent S-supersymmetry transformation, a field-dependent SU(2) R-symmetry transformation, and a field-dependent U(1) chiral transformation,

$$
\left.\delta_{\mathrm{Q}}(\epsilon)\right|_{5 D} ^{\mathrm{reduced}} \Phi=\left.\delta_{\mathrm{Q}}(\epsilon)\right|_{4 D} \Phi+\left.\delta_{\mathrm{S}}(\tilde{\eta})\right|_{4 D} \Phi+\left.\delta_{\mathrm{SU}(2)}(\tilde{\Lambda})\right|_{4 D} \Phi+\left.\delta_{\mathrm{U}(1)}\left(\tilde{\Lambda}^{0}\right)\right|_{4 D} \Phi .
$$


To give a meaning to the right-hand side one has to identify fields $\Phi$ that transform covariantly in the $4 D$ setting, so that all transformations in the above decomposition are clearly defined. The identification of these fields is done iteratively. Here one has to realize that the $5 D$ transformations for the Weyl multiplet are defined in a background consisting of the $5 D$ Weyl multiplet, whereas the $4 D$ transformations of the matter multiplets are defined in the $4 D$ background. But the field-dependent parameters in (2.14) are not restricted and still depend on a variety of the $5 D$ Weyl multiplet fields. These parameters, $\tilde{\eta}^{i}, \tilde{\Lambda}$ and $\tilde{\Lambda}^{0}$, are defined as follows (consistent with the lowest-order contributions that we have already exhibited in (2.13)),

$$
\begin{aligned}
\tilde{\eta}^{i}= & \frac{1}{3} A \gamma^{5} \epsilon^{i}+\frac{1}{8} \mathrm{i} \gamma^{5} \phi^{-1}\left(\hat{F}(B)_{a b}-4 \mathrm{i} \phi T_{a b} \gamma_{5}\right) \gamma^{a b} \epsilon^{i} \\
& +\frac{1}{4} \mathrm{i} \phi^{2}\left(\bar{\psi}_{j} \gamma^{5} \psi^{i} \gamma_{5}-\bar{\psi}_{j} \psi^{i}+\bar{\psi}_{j} \gamma^{a} \psi^{i} \gamma_{a}+\frac{1}{2} \bar{\psi}_{k} \gamma^{5} \gamma^{a} \psi^{k} \gamma_{5} \gamma_{a} \delta_{j}{ }^{i}\right) \epsilon^{j}, \\
\tilde{\Lambda}_{i}^{j}= & \phi\left(\bar{\epsilon}_{k} \gamma^{5} \psi^{l} \varepsilon_{i l} \varepsilon^{j k}-\frac{1}{2} \bar{\epsilon}_{k} \gamma^{5} \psi^{k} \delta_{i}^{j}\right), \\
\tilde{\Lambda}^{0}= & \mathrm{i} \phi \bar{\epsilon}_{i} \psi^{i} .
\end{aligned}
$$

Let us briefly discuss the non-linear corrections to (2.13), whose contributions were already included in (2.15). They originate from three sources, namely the compensating Lorentz transformation (2.7), the non-supercovariant term in the spin connection $\omega_{\mu}{ }^{a 5}$, and the non-linearity in the definition of the $4 D$ gravitini $\psi_{\mu}^{i}$ in terms of the $5 D$ fields (c.f. (2.8)). Concentrating on variations that explicitly contain $\psi_{\mu}{ }^{i}$, one easily notes that they no longer satisfy the standard supercovariance properties (which are manifest in four and five dimensions). In principle it is possible to absorb some of the unwanted terms in some of the bosonic fields appearing on the right-hand side of (2.13) or in the fielddependent S-transformations. The only fields, however, that can accommodate terms proportional to the bare (i.e. not contained in covariant objects) gravitini, are the R-symmetry gauge fields $V_{\mu i}{ }^{j}$. However, in that case the supersymmetry variation of these gauge fields will acquire terms proportional to derivatives on the supersymmetry parameter $\epsilon^{i}$, which can only be interpreted as an extra field-dependent $\mathrm{SU}(2)$ R-symmetry transformations, as is already indicated in (2.14). However, there are also higher-order variations proportional to $\psi^{i}$, so the situation becomes considerably more involved. In deciding how to deal with all these terms, some guidance can be obtained from reducing, at the same time, the matter multiplets. But for the sake of clarity, we prefer not to mix the presentation of the Weyl multiplet reduction with the presentation of the reduction of the matter multiplets. The latter will therefore be postponed to section 3. At this point we will simply take note this extra evidence and restrict our discussion here to the Weyl multiplet reduction. 
The result of the reduction motivates the following redefinitions of the various fields,

$$
\begin{aligned}
\hat{A}_{\mu} & =A_{a} e_{\mu}{ }^{a}-\frac{1}{2} \mathrm{i} \phi \bar{\psi}_{j} \psi_{\mu}{ }^{j}-\frac{1}{4} \mathrm{i} \phi^{2} \bar{\psi}_{j} \gamma^{5} \gamma_{\mu} \psi^{j}, \\
\hat{T}_{a b} & =24 T_{a b}+\mathrm{i} \phi^{-1} \varepsilon_{a b c d} \hat{F}(B)^{c d}-\mathrm{i} \phi^{2} \bar{\psi}_{i} \gamma_{a b} \psi^{i}, \\
\hat{V}_{j}{ }^{i} & =\phi^{2}\left(V_{j}{ }^{i}-\frac{3}{2} \phi \bar{\psi}_{j} \gamma^{5} \psi^{i}\right), \\
\hat{V}_{\mu j}{ }^{i} & =V_{\mu j}{ }^{i}-\phi\left(\bar{\psi}_{\mu j} \gamma^{5} \psi^{i}-\frac{1}{2} \delta_{j}{ }^{i} \bar{\psi}_{\mu k} \gamma^{5} \psi^{k}\right)-\frac{1}{2} \phi^{2} \bar{\psi}_{j} \gamma_{\mu} \psi^{i} .
\end{aligned}
$$

These are the linear combinations that emerge in the $4 D$ supersymmetry transformations. Their S-supersymmetry transformations turn out to be relevant and we note the following result,

$$
\begin{aligned}
\delta \hat{A}_{\mu} & =\frac{1}{2} \bar{\psi}_{\mu j} \gamma^{5} \eta^{j} \\
\delta \hat{T}_{a b} & =0 \\
\delta \hat{V}_{j}^{i} & =0 \\
\delta \hat{V}_{\mu j}{ }^{i} & =-2 \mathrm{i}\left(\bar{\psi}_{\mu j} \eta^{i}-\frac{1}{2} \delta_{j}{ }^{i} \bar{\psi}_{\mu k} \eta^{k}\right) .
\end{aligned}
$$

In particular, note that the factor in the variation of $\hat{V}_{\mu i}{ }^{j}$ has now changed as compared to the factor that appears in the corresponding $5 D$ S-variation given in (2.1). Furthermore, note that $\hat{A}_{\mu}$ is not supercovariant; its Q-supersymmetry variation contains a term proportional to the derivative of the supersymmetry parameter. Eventually $\hat{A}_{\mu}$ will be related to a gauge field associated with the $4 D \mathrm{U}(1) \mathrm{R}$-symmetry. This is consistent with the fact that $\hat{A}_{\mu}$ transforms into the gravitino fields under S-supersymmetry, in agreement with $4 D$ results.

With these notational changes we repeat the Q-supersymmetry transformations of (2.9) and (2.13), including also the higher-order contributions. First we consider those associated with the Kaluza-Klein vector multiplet,

$$
\begin{aligned}
\delta \phi= & -\phi^{2} \bar{\epsilon}_{i} \gamma^{5} \psi^{i}, \\
\delta B_{\mu}= & \phi^{2} \bar{\epsilon}_{i} \gamma_{\mu} \psi^{i}+\phi \bar{\epsilon}_{i} \gamma^{5} \psi_{\mu}{ }^{i}, \\
\delta\left(\phi^{2} \psi^{i}\right)= & -\frac{1}{2}\left[\hat{F}(B)_{a b}-\frac{1}{8} \mathrm{i} \gamma_{5} \phi \hat{T}_{a b}\right] \gamma^{a b} \epsilon^{i} \\
& -\left[\left(\partial_{\mu}-b_{\mu}\right) \phi \gamma^{5}+\frac{1}{2} \phi^{2}\left(\bar{\psi}_{\mu j} \gamma^{5} \psi^{j} \gamma^{5}+\bar{\psi}_{\mu j} \psi^{j}\right)+\mathrm{i} \phi \hat{A}_{\mu}\right] \gamma^{\mu} \epsilon^{i} \\
& +\hat{V}_{j}{ }^{i} \epsilon^{j}-\frac{1}{2} \mathrm{i} \tilde{\Lambda}^{0} \phi^{2} \gamma^{5} \psi^{i}-\mathrm{i} \phi \gamma^{5} \eta^{i},
\end{aligned}
$$

where here and henceforth we suppress the S-supersymmetry and R-symmetry transformations proportional to the field-dependent parameters $\tilde{\eta}^{i}$ and $\tilde{\Lambda}_{i}{ }^{j}$. However, we did include 
the $\mathrm{U}(1)$ transformation with parameter $\tilde{\Lambda}^{0}$, just as in the next formula. Apart from some minor details, these variations show the same structure as the $4 D$ transformation rules of vector multiplet, except that we have been unable to identify the second scalar field. The field $\hat{V}_{j}^{i}$ is obviously related to the auxiliary field of this vector multiplet.

The Q-supersymmetry transformations of the Weyl multiplet fields are as follows,

$$
\begin{aligned}
\delta e_{\mu}{ }^{a}= & \bar{\epsilon}_{i} \gamma^{a} \psi_{\mu}{ }^{i}, \\
\delta \psi_{\mu}{ }^{i}= & 2\left(\partial_{\mu}-\frac{1}{4} \omega_{\mu}{ }^{a b} \gamma_{a b}+\frac{1}{2} b_{\mu}+\frac{1}{2} \mathrm{i} \hat{A}_{\mu} \gamma_{5}\right) \epsilon^{i}+\hat{V}_{\mu j}{ }^{i} \epsilon^{j} \\
& +\frac{1}{16} \mathrm{i} \hat{T}_{a b} \gamma^{a b} \gamma_{\mu} \epsilon^{i}-\frac{1}{2} \mathrm{i} \tilde{\Lambda}^{0} \gamma^{5} \psi_{\mu}{ }^{i}-\mathrm{i} \gamma_{\mu} \eta^{i},
\end{aligned}
$$

Also in this case the variations show a close resemblance to the $4 D$ transformation rules of the $4 D$ Weyl multiplet fields, with $\hat{V}_{\mu j}{ }^{i}$ playing the role of the $\mathrm{SU}(2)$ gauge fields. In both the above results $\hat{A}_{\mu}$ seems to play the role of the $\mathrm{U}(1)$ chiral gauge field, and $\hat{T}_{a b}$ is the $4 D$ tensor field.

We now return to the issue of the missing spinless field in the Kaluza-Klein vector multiplet. The crucial observation is that the expressions obtained so far are consistent with the assumption that we are dealing with a gauge-fixed version of the theory. So we simply have to introduce a phase for the vector multiplet scalar which transforms locally under $\mathrm{U}(1)$ transformations. This is achieved by introducing the following R-covariant spinors, transforming under local $\mathrm{SU}(2) \times \mathrm{U}(1)$ R-symmetry transformations,

$$
\begin{aligned}
\left.\epsilon^{i}\right|^{\text {Rcov }} & =\exp \left[-\frac{1}{2} \mathrm{i} \varphi \gamma^{5}\right] \epsilon^{i}, \\
\left.\eta^{i}\right|^{\text {Rcov }} & =\exp \left[\frac{1}{2} \mathrm{i} \varphi \gamma^{5}\right] \eta^{i}, \\
\left.\psi_{\mu}{ }^{i}\right|^{\text {Rcov }} & =\exp \left[-\frac{1}{2} \mathrm{i} \varphi \gamma^{5}\right] \psi_{\mu}^{i}, \\
\left.\psi^{i}\right|^{\text {Rcov }} & =\exp \left[-\frac{1}{2} \mathrm{i} \varphi \gamma^{5}\right] \psi^{i}
\end{aligned}
$$

and assume that the phase factor $\varphi$ transforms under supersymmetry and under a new local $\mathrm{U}(1)$ group according to

$$
\delta \varphi=\Lambda^{0}-\mathrm{i} \phi \bar{\epsilon}_{i} \psi^{i},
$$

where $\Lambda^{0}$ is now an arbitrary space-time dependent function. Imposing a U(1) gauge choice $\varphi=0$ then generates a compensating $\mathrm{U}(1)$ component in the Q-variations, so that these terms re-emerge in the supersymmetry transformations for the fermions. The R-covariant spinors are not yet converted to the standard chiral $4 D$ spinors, but possess already all the necessary features

The variations (2.18) can now be rewritten in terms of R-covariant spinors. Here and henceforth we will suppress the superscript Rcov. Furthermore we employ chiral spinors 
defined by $\gamma^{5} \psi_{ \pm}= \pm \psi_{ \pm}$. The result takes the form,

$$
\begin{aligned}
\delta\left(\mathrm{e}^{\mp \mathrm{i} \varphi} \phi\right)= & \mp 2 \phi^{2} \bar{\epsilon}_{i} \psi_{ \pm}^{i}, \\
\delta B_{\mu}= & \bar{\epsilon}_{i}\left[\gamma_{\mu} \phi^{2} \psi_{-}^{i}+\phi \mathrm{e}^{\mathrm{i} \varphi} \psi_{\mu+}^{i}\right]+\bar{\epsilon}_{i}\left[\gamma_{\mu} \phi^{2} \psi_{+}^{i}-\phi \mathrm{e}^{-\mathrm{i} \varphi} \psi_{\mu-}^{i}\right], \\
\delta\left(\phi^{2} \psi_{ \pm}^{i}\right)= & -\frac{1}{2}\left[\hat{F}(B)_{a b} \mp \frac{1}{8} \mathrm{i} \phi \hat{T}_{a b}\right] \gamma^{a b} \epsilon_{ \pm}^{i} \\
& \mp \not D\left(\phi \mathrm{e}^{\mp \mathrm{i} \varphi}\right) \epsilon_{\mp}^{i}+\hat{V}_{j}^{i} \epsilon_{ \pm}^{j} \mp \mathrm{i} \phi \mathrm{e}^{\mp \mathrm{i} \varphi} \eta_{ \pm}^{i},
\end{aligned}
$$

where $\hat{D}_{\mu}\left(\phi \mathrm{e}^{\mp \mathrm{i} \varphi}\right)$ is a supercovariant and $\mathrm{U}(1)$ covariant derivative, defined by

$$
\hat{D}_{\mu}\left(\phi \mathrm{e}^{\mp \mathrm{i} \varphi}\right)=\left(\partial_{\mu}-b_{\mu}\right)\left(\phi \mathrm{e}^{\mp \mathrm{i} \varphi}\right) \pm \phi^{2} \bar{\psi}_{\mu i} \psi_{ \pm}^{i} \pm \mathrm{i} A_{\mu}\left(\phi \mathrm{e}^{\mp \mathrm{i} \varphi}\right) .
$$

Here the $\mathrm{U}(1)$ connection equals

$$
A_{\mu}=\hat{A}_{\mu}+\partial_{\mu} \varphi
$$

Hence the R-symmetry group has now been extended to $\mathrm{SU}(2) \times \mathrm{U}(1)$. Observe that $A_{\mu}$ transforms covariantly under supersymmetry. The definition (2.24) can be written in supercovariant form,

$$
\hat{D}_{\mu} \varphi=-6 T_{a 5} e_{\mu}{ }^{a}+\frac{1}{4} \phi^{2} \bar{\psi}_{i} \gamma^{5} \gamma_{\mu} \psi^{i} .
$$

The same manipulations can be applied to the fields of the Weyl multiplet, and we give the result for the vielbein and gravitino fields, the latter again written with $\mathrm{R}$ covariant spinors,

$$
\begin{aligned}
\delta e_{\mu}{ }^{a}= & \bar{\epsilon}_{i} \gamma^{a} \psi_{\mu+}^{i}+\bar{\epsilon}_{i} \gamma^{a} \psi_{\mu-}^{i}, \\
\delta \psi_{\mu \pm}^{i}= & 2\left(\partial_{\mu}-\frac{1}{4} \omega_{\mu}^{a b} \gamma_{a b}+\frac{1}{2} b_{\mu} \pm \frac{1}{2} \mathrm{i} A_{\mu}\right) \epsilon_{ \pm}^{i}+\hat{V}_{\mu j}{ }^{i} \epsilon_{ \pm}^{j} \\
& +\frac{1}{16} \mathrm{ie}^{\mp \mathrm{i} \varphi} \hat{T}_{a b} \gamma^{a b} \gamma_{\mu} \epsilon_{\mp}^{i}-\mathrm{i} \gamma_{\mu} \eta_{\mp}^{i},
\end{aligned}
$$

Apart from different spinor conventions and normalizations the supersymmetry variations take the same form as the corresponding ones in four dimensions. There is a subtlety, which is that the $4 D$ tensor field can be split in selfdual and anti-selfdual components and should be identified with $\mathrm{e}^{ \pm \mathrm{i} \varphi} \hat{T}_{a b}{ }^{ \pm}$, where the superscript \pm on the tensor indicates its duality phase. After this identification the correspondence between the U(1) and the chirality/duality assignments is precisely as in the four-dimensional theory. The Kaluza-Klein multiplet transforms as a proper vector supermultiplet in a $4 D$ superconformal background. Its auxiliary field $\hat{V}_{i}^{j}$ is indeed an $\mathrm{SU}(2)$ vector. As a check we have verified that its transformations take the form expected from the $4 D$ transformation.

For the Weyl multiplet, we established that the vielbein and the gravitino transformations are also in line with the $4 D$ transformations. We have already obtained the correct expressions of the R-symmetry gauge fields. The transformations of these fields will lead to 
rather complicated expressions that include also the constrained gauge fields. However, the constraints have a different form in four and in five dimensions which is related to certain field redefinitions, and this must be taken into account when comparing. As we have mentioned above, we have already identified the $4 D$ auxiliary tensor field, and likewise we can deduce the expressions for the $5 D$ fields $\chi^{i}$ and $D$ from the explicit variations in terms of the $4 D$ fields. The corresponding formulae will be presented in (4.5). Some of these results are convenient when comparing $4 D$ and $5 D$ actions related by dimensional reduction.

The final result of this section is that the off-shell dimensional reduction of $5 D$ multiplets in a superconformal background can be carried out systematically. The transformations originating from the $5 \mathrm{D}$ multiplets are identical to those in $4 D$ up to field-dependent S-supersymmetry and R-symmetry transformations. This makes the actual identification of the proper $4 D$ fields non-trivial. The resulting $4 D$ theory can understood as a gaugefixed version of the standard theory. The gauge-fixing is related to the extra R-symmetry that arises in lower dimensions. Both these features are generic.

\section{Off-shell dimensional reduction; matter multiplets}

In this section we repeat the same analysis as in the previous section, but now applied to the vector multiplet and the hypermultiplet. We refrain from presenting similar results for tensor multiplets. They can be derived by the same method, or, alternatively, they can be found by considering a composite tensor multiplet constructed from the square of a vector multiplet.

In five space-time dimensions the vector supermultiplet consists of a real scalar $\sigma$, a gauge field $W_{\mu}$, a triplet of (auxiliary) fields $Y^{i j}$, and a fermion field $\Omega^{i}$. Under Q- and S-supersymmetry these fields transform as follows,

$$
\begin{aligned}
\delta \sigma & =\mathrm{i} \bar{\epsilon}_{i} \Omega^{i} \\
\delta \Omega^{i} & =-\frac{1}{2}\left(\hat{F}_{A B}-4 \sigma T_{A B}\right) \gamma^{A B} \epsilon^{i}-\mathrm{i} \not D \sigma \epsilon^{i}-2 \varepsilon_{j k} Y^{i j} \epsilon^{k}+\sigma \eta^{i}, \\
\delta W_{M} & =\bar{\epsilon}_{i} \gamma_{M} \Omega^{i}-\mathrm{i} \sigma \bar{\epsilon}_{i} \psi_{M}^{i} \\
\delta Y^{i j} & =\varepsilon^{k(i} \bar{\epsilon}_{k} \not D \Omega^{j)}+2 \mathrm{i} \varepsilon^{k(i} \bar{\epsilon}_{k}\left(-\frac{1}{4} T_{A B} \gamma^{A B} \Omega^{j)}+4 \sigma \chi^{j)}\right)-\frac{1}{2} \mathrm{i} \varepsilon^{k(i} \bar{\eta}_{k} \Omega^{j)}
\end{aligned}
$$

where $\left(Y^{i j}\right)^{*} \equiv Y_{i j}=\varepsilon_{i k} \varepsilon_{j l} Y^{k l}$, and the supercovariant field strength is defined as,

$$
\hat{F}_{M N}(W)=2 \partial_{[M} W_{N]}-\bar{\Omega}_{i} \gamma_{[M} \psi_{N]}^{i}+\frac{1}{2} \mathrm{i} \sigma \bar{\psi}_{[M i} \psi_{N]}^{i} .
$$

The fields behave under local scale transformations according to the weights shown in table 2 .

The reduction proceeds in the same way as before, except that we have now the advantage that we have already identified some of the $4 D$ fields belonging to the $4 D$ Weyl 


\begin{tabular}{|c|cccc|cc|}
\hline & \multicolumn{4}{|c|}{ vector multiplet } & \multicolumn{2}{|c|}{ hypermultiplet } \\
\hline field & $\sigma$ & $W_{\mu}$ & $\Omega_{i}$ & $Y_{i j}$ & $A_{i}{ }^{\alpha}$ & $\zeta^{\alpha}$ \\
\hline$w$ & 1 & 0 & $\frac{3}{2}$ & 2 & $\frac{3}{2}$ & 2 \\
\hline
\end{tabular}

Table 2. Weyl weights $w$ of the vector multiplet and the hypermultiplet component fields in five space-time dimensions.

multiplet. We decompose the $5 D$ gauge field $W_{M}$ into a four-dimensional gauge field $W_{\mu}$ and a scalar $W=W_{5}$ by using the standard Kaluza-Klein ansatz, and write the Q- and S-transformation rules, including the compensating Lorentz transformation (2.7). Just as in (2.20) we introduce an R-covariant spinor field field $\Omega^{i}$ by

$$
\left.\left(\Omega^{i}-\phi^{2} W \psi^{i}\right)\right|^{\mathrm{Rcov}}=\exp \left[-\frac{1}{2} \mathrm{i} \varphi \gamma^{5}\right]\left(\Omega^{i}-\phi^{2} W \psi^{i}\right),
$$

which transforms under $\mathrm{U}(1)$. In terms of the R-covariant spinor fields, we derive the following transformation rules,

$$
\begin{aligned}
\delta\left[\mathrm{e}^{\mp \mathrm{i} \varphi}(\sigma \pm \mathrm{i} \phi W)\right]= & 2 \mathrm{i} \bar{\epsilon}_{i}\left(\Omega^{i}-\phi^{2} W \psi^{i}\right)_{ \pm}, \\
\delta W_{\mu}= & \bar{\epsilon}_{i}\left[\gamma_{\mu}\left(\Omega^{i}-\phi^{2} W \psi^{i}\right)_{-}-\mathrm{i}(\sigma-\mathrm{i} \phi W) \mathrm{e}^{\mathrm{i} \varphi} \psi_{\mu}^{i}+\right] \\
& +\bar{\epsilon}_{i}\left[\gamma_{\mu}\left(\Omega^{i}-\phi^{2} W \psi^{i}\right)_{+}-\mathrm{i}(\sigma+\mathrm{i} \phi W) \mathrm{e}^{-\mathrm{i} \varphi} \psi_{\mu}{ }^{i}-\right] \\
\delta\left(\Omega^{i}-\phi^{2} W \psi^{i}\right)_{ \pm}= & -\frac{1}{2}\left[\hat{F}(W)_{a b}-\frac{1}{8}(\sigma \mp \mathrm{i} \phi W) \hat{T}_{a b}\right] \gamma^{a b} \epsilon_{ \pm}^{i} \\
& -\mathrm{i} \hat{D}\left[(\sigma \pm \mathrm{i} \phi W) \mathrm{e}^{\mp \mathrm{i} \varphi}\right] \epsilon_{\mp}^{i}-2 \hat{Y}^{i k} \varepsilon_{k j} \epsilon_{ \pm}^{j} \\
& +(\sigma \pm \mathrm{i} \phi W) \mathrm{e}^{\mp \mathrm{i} \varphi} \eta_{ \pm}^{i},
\end{aligned}
$$

where $\hat{Y}^{i j}$ is defined by

$$
\hat{Y}^{i j}=Y^{i j}+\frac{1}{2} W \hat{V}_{k}^{i} \varepsilon^{j k}+\frac{1}{2} \phi\left(\bar{\Omega}_{k} \gamma^{5}-\frac{1}{2} \mathrm{i} \sigma \phi \bar{\psi}_{k}\right) \psi^{(i} \varepsilon^{j) k} .
$$

Note that in (3.4), we have again suppressed the field-dependent S-supersymmetry and $\mathrm{SU}(2)$ R-symmetry transformations.

Hypermultiplets are associated with target spaces of dimension $4 r$ that are hyperkähler cones [14]. The supersymmetry transformations are most conveniently written in terms of the sections $A_{i}{ }^{\alpha}(\phi)$, where $\alpha=1,2, \ldots, 2 r$,

$$
\begin{aligned}
\delta A_{i}{ }^{\alpha} & =2 \mathrm{i} \bar{\epsilon}_{i} \zeta^{\alpha}, \\
\delta \zeta^{\alpha} & =-\mathrm{i} \not \supset A_{i}{ }^{\alpha} \epsilon^{i}+\frac{3}{2} A_{i}{ }^{\alpha} \eta^{i} .
\end{aligned}
$$

The $A_{i}{ }^{\alpha}$ are the local sections of an $\operatorname{Sp}(r) \times \operatorname{Sp}(1)$ bundle. We also note the existence of a covariantly constant skew-symmetric tensor $\Omega_{\alpha \beta}$ (and its complex conjugate $\Omega^{\alpha \beta}$ satisfying $\Omega_{\alpha \gamma} \Omega^{\beta \gamma}=-\delta_{\alpha}{ }^{\beta}$ ), and the symplectic Majorana condition for the spinors reads as 


\begin{tabular}{|c|c|c|c|c|c|c|}
\hline & \multicolumn{4}{|c|}{ vector multiplet } & \multicolumn{2}{|c|}{ hypermultiplet } \\
\hline field & $X$ & $W_{\mu}$ & $\Omega_{i}$ & $Y^{i j}$ & $A_{i}^{\alpha}$ & $\zeta^{\alpha}$ \\
\hline$w$ & 1 & 0 & $\frac{3}{2}$ & 2 & 1 & $\frac{3}{2}$ \\
\hline$c$ & -1 & 0 & $-\frac{1}{2}$ & 0 & 0 & $-\frac{1}{2}$ \\
\hline$\gamma_{5}$ & & & + & & & - \\
\hline
\end{tabular}

Table 3. Weyl and chiral weights $\left(w\right.$ and $c$ ) and fermion chirality $\left(\gamma_{5}\right)$ of the vector multiplet and the hypermultiplet component fields in four space-time dimensions.

$C^{-1} \bar{\zeta}_{\alpha}^{\mathrm{T}}=\Omega_{\alpha \beta} \zeta^{\beta}$. Covariant derivatives contain the $\operatorname{Sp}(r)$ connection $\Gamma_{A}{ }_{\beta}{ }_{\beta}$, associated with rotations of the fermions. The sections $A_{i}{ }^{\alpha}$ are pseudo-real, i.e. they are subject to the constraint, $A_{i}{ }^{\alpha} \varepsilon^{i j} \Omega_{\alpha \beta}=A_{\beta}^{j} \equiv\left(A_{j}{ }^{\beta}\right)^{*}$. The information on the target-space metric is contained in the so-called hyperkähler potential. For our purpose the geometry of the hyperkähler cone is not relevant. Hence we assume that the cone is flat, so that the targetspace connections and curvatures will vanish. The extension to non-trivial hyperkähler cone geometries is straightforward.

For the local scale transformations we refer again to the weights shown in table 2 . The hypermultiplet is not realized as an off-shell supermultiplet. Closure of the superconformal transformations is only realized upon using fermionic field equations, but this fact does not represent a serious problem in what follows. The $4 D$ fields have, however, different Weyl weights as is shown in table 3 . This has been taken into account in the reduction, by scaling $A_{i}{ }^{\alpha}$ by a factor $\phi^{-1 / 2}$, as can be seen below. Furthermore we define an R-covariant spinor combination,

$$
\left.\left(\phi^{-1 / 2} \zeta^{\alpha}-\frac{1}{2} \phi^{1 / 2} A_{j}{ }^{\alpha} \gamma^{5} \psi^{j}\right)\right|^{\text {Rcov }}=\exp \left[\frac{1}{2} \mathrm{i} \varphi \gamma^{5}\right]\left(\phi^{-1 / 2} \zeta^{\alpha}-\frac{1}{2} \phi^{1 / 2} A_{j}^{\alpha} \gamma^{5} \psi^{j}\right) .
$$

The $5 D$ Q- and S-supersymmetry variations take the following form, again in terms of R-covariant chiral spinors,

$$
\begin{aligned}
\delta\left(\phi^{-1 / 2} A_{i}{ }^{\alpha}\right)= & 2 \mathrm{i} \bar{\epsilon}_{i}\left(\phi^{-1 / 2} \zeta^{\alpha}-\frac{1}{2} \mathrm{i} \phi^{1 / 2} A_{j}{ }^{\alpha} \gamma^{5} \psi^{j}\right)_{+} \\
& +2 \mathrm{i} \bar{\epsilon}_{i}\left(\phi^{-1 / 2} \zeta^{\alpha}-\frac{1}{2} \mathrm{i} \phi^{1 / 2} A_{j}{ }^{\alpha} \gamma^{5} \psi^{j}\right)_{-} \\
\delta\left(\phi^{-1 / 2} \zeta^{\alpha}-\frac{1}{2} \mathrm{i} \phi^{1 / 2} A_{j}{ }^{\alpha} \gamma^{5} \psi^{j}\right)_{ \pm}= & -\mathrm{i} \not{D}\left(\phi^{-1 / 2} A_{i}^{\alpha}\right) \epsilon_{\mp}^{i}+\phi^{-1 / 2} A_{i}{ }^{\alpha} \eta_{ \pm}^{i}
\end{aligned}
$$

where, as before, we suppressed the S-supersymmetry and R-symmetry transformations with field-dependent parameters as specified by (2.15). Note that the proportionality factor in front of the $4 D$ S-supersymmetry variation has changed as compared to the $5 D$ result (3.6). 


\section{Five and four-dimensional fields and invariant Lagrangians}

After expressing the $5 D$ spinors into chiral $4 D$ spinors according to the procedure explained in appendix B, we can identify the $4 D$ fields in terms of the $5 D$ ones. Note that for matter fields the overall normalization of the components is only determined up to a real constant. For the vector multiplet we choose the normalization such that the vector gauge field remains the same. This has the advantage that we can easily compare the corresponding charges in four and five dimensions. Phase factors can be changed according to the chiral $\mathrm{U}(1)$ transformations which constitute an invariance of the theory, but they should be applied uniformly. Our four-dimensional transformations coincide with those given in [9]. For the convenience of the reader we have also included a summary in appendix C.

We thus express the $4 D$ fields in terms of the $5 D$ fields and the field $\varphi$ for each multiplet separately. First we present the Kaluza-Klein and the matter vector multiplets, then the hypermultiplet, and finally we turn to the Weyl multiplet.

The Kaluza-Klein vector multiplet:

$$
\begin{aligned}
X^{0} & =-\frac{1}{2} \phi \mathrm{e}^{-\mathrm{i} \varphi}, \\
\Omega_{i}{ }^{0} & =-\varepsilon_{i j} \phi^{2} \mathrm{e}^{-\frac{1}{2} \mathrm{i} \varphi} \psi_{+}^{j}, \quad \Omega^{i 0}=\mathrm{i} \phi^{2} \mathrm{e}^{\frac{1}{2} \mathrm{i} \varphi} \psi_{-}^{i}, \\
W_{\mu}{ }^{0} & =B_{\mu}, \\
Y^{i j 0} & =\hat{V}_{k}{ }^{i} \varepsilon^{j k},
\end{aligned}
$$

The matter vector multiplet:

$$
\begin{aligned}
X & =-\frac{1}{2} \mathrm{i}(\sigma+\mathrm{i} \phi W) \mathrm{e}^{-\mathrm{i} \varphi}, \\
\Omega_{i} & =-\varepsilon_{i j} \mathrm{e}^{-\frac{1}{2} \mathrm{i} \varphi}\left(\Omega^{j}-\phi^{2} W \psi^{j}\right)_{+}, \quad \Omega^{i}=\mathrm{ie}^{\frac{1}{2} \mathrm{i} \varphi}\left(\Omega^{i}-\phi^{2} W \psi^{i}\right)_{-}, \\
W_{\mu} & =W_{\mu}, \\
Y^{i j} & =-2 \hat{Y}^{i j},
\end{aligned}
$$

The hypermultiplet:

$$
\begin{aligned}
A_{i}^{\alpha} & =\phi^{-1 / 2} A_{i}^{\alpha}, \\
\zeta^{\alpha} & =\mathrm{e}^{-\frac{1}{2} \mathrm{i} \varphi}\left(\phi^{-1 / 2} \zeta^{\alpha}-\frac{1}{2} \phi^{1 / 2} A_{j}^{\alpha} \gamma^{5} \psi^{j}\right)_{-}, \\
\zeta_{\alpha} & =-\mathrm{i} \Omega_{\alpha \beta} \mathrm{e}^{\frac{1}{2} \mathrm{i} \varphi}\left(\phi^{-1 / 2} \zeta^{\beta}-\frac{1}{2} \phi^{1 / 2} A_{j}{ }^{5} \gamma^{5} \psi^{j}\right)_{+} .
\end{aligned}
$$

The Weyl multiplet:

$$
\begin{aligned}
& e_{\mu}{ }^{a}=e_{\mu}{ }^{a}, \\
& \psi_{\mu}{ }^{i}=\mathrm{e}^{-\frac{1}{2} \mathrm{i} \varphi} \psi_{\mu+}^{i},
\end{aligned} \quad \psi_{\mu i}=\mathrm{i} \varepsilon_{i j} \mathrm{e}^{\frac{1}{2} \mathrm{i} \varphi} \psi_{\mu_{-}}^{j},
$$




$$
\begin{aligned}
& T_{a b}{ }^{i j}=-\frac{1}{2} \mathrm{e}^{-\mathrm{i} \varphi} \hat{T}_{a b}^{-} \varepsilon^{i j}, \\
& \mathcal{V}_{\mu}{ }^{i}{ }_{j}=\hat{V}_{\mu j}{ }^{i}, \quad b_{\mu}=b_{\mu}, \quad A_{\mu}=\hat{A}_{\mu}+\partial_{\mu} \varphi .
\end{aligned}
$$

The remaining fermion fields of the $5 D$ Weyl multiplet, $\phi_{M}{ }^{i}$ and $\chi^{i}$, follow from the $5 D$ Q-supersymmetry transformations of $b_{M}$ and $V_{M i}{ }^{j}$. Likewise the remaining fermions of the $4 D$ Weyl multiplet, $\phi_{\mu}{ }^{i}$ and $\chi^{i}$, follow from the Q-supersymmetry variations of $b_{\mu}$ or $\mathcal{V}_{\mu j}{ }^{i}$, and $A_{\mu}$. To disentangle the two sets of fermion fields one makes use of the conventional constraints. The relevant $5 D$ constraint was given in (2.4) and the corresponding $4 D$ constraints are given in (C.3). The same comment applies to the composite gauge fields $f_{M}{ }^{A}$ and $f_{\mu}{ }^{a}$ corresponding to the $5 D$ and $4 D$ conformal boosts, respectively. Finally one determines the scalar field $D$ from considering the variation of the field $\chi^{i}$. We summarize some of the relevant results below, suppressing terms of higher order in the fermion fields,

$$
\begin{aligned}
\left.f_{a}{ }^{a}\right|_{4 D}= & \left.f_{a}{ }^{a}\right|_{5 D}-\left.\frac{1}{2} D\right|_{4 D}-\frac{1}{16} \phi^{-2} F(B)^{a b} F(B)_{a b} \\
\left.\phi_{\mu}{ }^{i}\right|_{4 D}= & \left.2 \mathrm{i} \phi_{\mu}{ }^{i}\right|_{5 D}+4 \gamma_{\mu} \chi^{i}+\left(e_{\mu}^{a}-\frac{3}{8} \gamma_{\mu} \gamma^{a}\right) \gamma_{5} \mathcal{D}_{a} \psi^{i}+\frac{3}{32} F(B)^{a b}\left(\gamma_{\mu} \gamma_{a b}-2 \gamma_{a b} \gamma_{\mu}\right) \psi^{i}, \\
& -\frac{3}{8} \mathrm{i} \phi T^{a b} \gamma^{5}\left(\gamma_{\mu} \gamma_{a b}-2 \gamma_{a b} \gamma_{\mu}\right) \psi^{i}-\frac{3}{8} \mathrm{i} \phi T_{a 5}\left(10 e_{\mu}{ }^{a}-\gamma^{a} \gamma_{\mu}\right) \psi^{i} \\
& -\frac{1}{2}\left(\not D \phi \phi \gamma_{\mu}+\frac{3}{2} \gamma_{\mu} \not D \phi\right) \gamma^{5} \psi^{i}-\frac{1}{4} \phi^{2} \mathcal{V}^{i}{ }_{j} \gamma_{\mu} \psi^{j} \\
\left.\chi^{i}\right|_{4 D}= & 8 \chi^{i}+\frac{1}{48} \gamma^{a b} F(B)_{a b} \psi^{i}-\frac{3}{4} \mathrm{i} \phi T_{a b} \gamma^{5} \gamma^{a b} \psi^{i}, \\
& +\frac{1}{4} \phi^{-1} \gamma_{5} \not D\left(\phi^{2} \psi^{i}\right)-\frac{1}{2} \phi^{2} \mathcal{V}^{i}{ }_{j} \psi^{j}-\frac{9}{4} \mathrm{i} \phi T_{a 5} \gamma^{a} \psi^{i} \\
\left.D\right|_{4 D}= & \left.4 D\right|_{5 D}+\frac{1}{4} \phi^{-1}\left(\mathcal{D}^{a} \mathcal{D}_{a}+\frac{1}{6} R\right) \phi+\frac{3}{32} \phi^{-2} F(B)^{a b} F(B)_{a b} \\
& +\frac{3}{2} T^{a b} T_{a b}+3 T^{a 5} T_{a 5}+\frac{1}{4} \phi^{2} V_{i}{ }^{j} V_{j}{ }^{i}
\end{aligned}
$$

where $D^{a} D_{a} \phi=\left(\mathcal{D}^{a} \mathcal{D}_{a}+\frac{1}{6} R\right) \phi$ equals the $4 D$ conformally invariant D'Alembertian with $R$ the $4 D$ Ricci scalar. One can proceed and rewrite the covariant derivatives on the spinors in terms of $4 D$ fields, to verify that the supersymmetry variations of the fields above are indeed identical to the ones in four dimensions, but this is not necessary here. The only result we will need in the remainder of this section is the last expression for the field $D$.

Suppressing the higher-order fermionic contributions we now express the $5 D$ bosonic fields into the $4 D$ ones. We assume that $\phi$ is positive so that we are considering compactification of a space-like coordinate. The $5 D$ components of the metric are already specified in (2.6). The remaining expressions are,

$$
\begin{aligned}
\phi & =2\left|X^{0}\right|, \\
B_{\mu} & =W_{\mu}{ }^{0},
\end{aligned}
$$




$$
\begin{aligned}
V_{M i}{ }^{j} & =\left\{\begin{array}{l}
V_{\mu i}{ }^{j}=\mathcal{V}_{\mu}{ }^{j}{ }_{i}-\frac{1}{4} \varepsilon_{i k} Y^{k j 0}\left|X^{0}\right|^{-2} W_{\mu}{ }^{0}, \\
V_{5 i}{ }^{j}=-\frac{1}{4} \varepsilon_{i k} Y^{k j 0}\left|X^{0}\right|^{-2},
\end{array}\right. \\
\sigma & =-\mathrm{i}\left|X^{0}\right|(t-\bar{t}), \\
W_{M} & =\left\{\begin{array}{l}
W_{\mu}=W_{\mu}-\frac{1}{2}(t+\bar{t}) W_{\mu}{ }^{0}, \\
W_{5}=-\frac{1}{2}(t+\bar{t}),
\end{array}\right. \\
Y^{i j} & =-\frac{1}{2} Y^{i j}+\frac{1}{4}(t+\bar{t}) Y^{i j 0}, \\
T_{a 5}= & \frac{1}{12} \mathrm{i} e_{a}{ }^{\mu}\left(\frac{\mathcal{D}_{\mu} X^{0}}{X^{0}}-\frac{\mathcal{D}_{\mu} \bar{X}^{0}}{\bar{X}^{0}}\right), \\
T_{a b}= & -\frac{\mathrm{i}}{24\left|X^{0}\right|}\left(\varepsilon_{i j} T_{a b}{ }^{i j} \bar{X}^{0}-F_{a b}^{-0}\right)+\text { h.c. },
\end{aligned}
$$

where $\mathcal{D}_{\mu} X^{0}=\left(\partial_{\mu}-b_{\mu}+\mathrm{i} A_{\mu}\right) X^{0}$ and $t=X / X^{0}$, and all the fields on the right-hand side refer to $4 D$ fields.

In the remainder of this section we evaluate the reduction of $5 D$ supersymmetric actions to four dimensions. We concentrate on actions for vector multiplets and for hypermultiplets, both at most quadratic in derivatives, and on a third action that contains terms proportional to the square of the Riemann tensor accompanied by other terms quartic in derivatives. In four dimensions, four different invariant actions are expected to be generated, related to the fact that there exist a second class of actions with higher-derivative couplings associated with the vector multiplets (see, e.g. [9] and references quoted therein). However, what we will establish below, is that there exists yet another higher-derivative action that involves terms quadratic in the Ricci tensor. This action has not appeared in the literature so far.

The $5 D$ bosonic Lagrangian for hypermultiplets reads

$$
8 \pi \mathcal{L}_{\text {hyper }}=-\frac{1}{2} E \Omega_{\alpha \beta} \varepsilon^{i j}\left\{\mathcal{D}_{M} A_{i}{ }^{\alpha} \mathcal{D}^{M} A_{j}{ }^{\beta}-A_{i}{ }^{\alpha} A_{j}{ }^{\beta}\left[\frac{3}{16} R+2 D+\frac{3}{4} T^{A B} T_{A B}\right]\right\} .
$$

Upon reduction to four dimensions, the first term becomes

$$
\begin{aligned}
-\frac{1}{2} E \Omega_{\alpha \beta} \varepsilon^{i j} \mathcal{D}_{M} A_{i}{ }^{\alpha} \mathcal{D}^{M} A_{j}{ }^{\beta}= & -\frac{1}{2} e \Omega_{\alpha \beta} \varepsilon^{i j}\left\{\mathcal{D}_{M} A_{i}{ }^{\alpha} \mathcal{D}^{M} A_{j}{ }^{\beta}\right. \\
& \left.+A_{i}{ }^{\alpha} A_{j}{ }^{\beta}\left[-\frac{1}{2} \mathcal{D}^{\mu}\left[\phi^{-1} \mathcal{D}_{\mu} \phi\right]+\frac{1}{4} \phi^{-2}\left[\mathcal{D}_{\mu} \phi\right]^{2}-\frac{1}{8} \phi^{2} V_{k}{ }^{l} V_{l}{ }^{k}\right]\right\},
\end{aligned}
$$

where we suppressed a total derivative. Next we turn to the second term in (4.7). Making use of (A.3), which relates the $5 D$ and $4 D$ Ricci scalars, and the relation between the $4 D$ and $5 D D$-fields, the combination of the two terms readily combines into

$$
8 \pi^{2} e^{-1} \mathcal{L}_{\text {hyper }}=-\frac{1}{2} \phi^{-1} \Omega_{\alpha \beta} \varepsilon^{i j}\left\{\mathcal{D}_{\mu} A_{i}{ }^{\alpha} \mathcal{D}^{\mu} A_{j}{ }^{\beta}-A_{i}{ }^{\alpha} A_{j}{ }^{\beta}\left(\frac{1}{2} D+\frac{1}{6} R\right)\right\},
$$


which agrees with the well-known expression for the supersymmetric $4 D$ Lagrangian [15]. Observe that the Kaluza-Klein vector multiplet decouples from the hypermultiplets, as it should.

Subsequently we turn to the $5 D$ bosonic Lagrangian for vector multiplets, whose evaluation is somewhat more cumbersome,

$$
\begin{aligned}
8 \pi^{2} \mathcal{L}_{\mathrm{vvv}}= & 3 E C_{A B C} \sigma^{A}\left[\frac{1}{2} \mathcal{D}_{M} \sigma^{B} \mathcal{D}^{M} \sigma^{C}+\frac{1}{4} F_{M N}{ }^{B} F^{M N C}-Y_{i j}{ }^{B} Y^{i j C}-3 \sigma^{B} F_{M N}{ }^{C} T^{M N}\right] \\
& -\frac{1}{8} \mathrm{i} C_{A B C} \varepsilon^{M N P P Q R} W_{M}{ }^{A} F_{N P}{ }^{B} F_{Q R}{ }^{C} \\
& -E C_{A B C} \sigma^{A} \sigma^{B} \sigma^{C}\left[\frac{1}{8} R-4 D-\frac{39}{2} T^{A B} T_{A B}\right]
\end{aligned}
$$

The first term is rewritten as,

$$
\begin{aligned}
3 E & C_{A B C} \sigma^{A}\left[\frac{1}{2} \mathcal{D}_{M} \sigma^{B} \mathcal{D}^{M} \sigma^{C}+\frac{1}{4} F_{M N}{ }^{B} F^{M N C}-Y_{i j}{ }^{B} Y^{i j C}-3 \sigma^{B} F_{M N}{ }^{C} T^{M N}\right]= \\
& -3 \mathrm{i} e\left|X^{0}\right|^{2} C_{A B C}(t-\bar{t})^{A} \mathcal{D}^{\mu} t^{B} \mathcal{D}_{\mu} \bar{t}^{C} \\
& +\frac{3}{4} \mathrm{i} e C_{A B C}(t-\bar{t})^{A}(t-\bar{t})^{B}(t-\bar{t})^{C}\left(\mathcal{D}_{\mu}\left|X^{0}\right|\right)^{2} \\
& -\frac{3}{2} \mathrm{i} e C_{A B C}(t-\bar{t})^{A}(t-\bar{t})^{B}\left(\bar{X}^{0} \mathcal{D}^{\mu} \bar{t}^{C} \mathcal{D}_{\mu} X^{0}-X^{0} \mathcal{D}^{\mu} t^{C} \mathcal{D}_{\mu} \bar{X}^{0}\right) \\
& -\frac{3}{8} \mathrm{i} e C_{A B C}(t-\bar{t})^{A}\left[F_{a b}{ }^{B} F^{a b C}-F_{a b}{ }^{B} F^{a b 0}(t+\bar{t})^{C}+\frac{1}{4}\left(F_{a b}\right)^{2}(t+\bar{t})^{B}(t+\bar{t})^{C}\right] \\
& +\frac{3}{8} \mathrm{i} e C_{A B C}(t-\bar{t})^{A}\left[Y_{i j}{ }^{B} Y^{i j C}-(t+\bar{t})^{B} Y_{i j}{ }^{C} Y^{i j 0}+\frac{1}{4}(t+\bar{t})^{B}(t+\bar{t})^{C}\left|Y_{i j}{ }^{0}\right|^{2}\right] \\
& -\frac{3}{16} \mathrm{i} e C_{A B C}(t-\bar{t})^{A}(t-\bar{t})^{B}\left[\left(F_{a b}{ }^{C}-\frac{1}{2}(t+\bar{t})^{C} F_{a b}{ }^{0}\right)\left(\varepsilon_{i j} T^{a b i j} \bar{X}^{0}-F^{-a b 0}\right)-\text { h.c. }\right],
\end{aligned}
$$

where we employed special coordinates $t^{A}=X^{A} / X^{0}$. The $5 D$ Chern-Simons term can be rewritten as follows,

$$
\begin{gathered}
-\frac{1}{8} \mathrm{i} C_{A B C} \varepsilon^{M N P Q R} W_{M}{ }^{A} F_{N P}{ }^{B} F_{Q R}{ }^{C}=\frac{1}{64} \mathrm{i} C_{A B C} \varepsilon^{\mu \nu \rho \sigma}\left[12(t+\bar{t})^{A} F_{\mu \nu}{ }^{B} F_{\rho \sigma}{ }^{C}\right. \\
\left.-6(t+\bar{t})^{A}(t+\bar{t})^{B} F_{\mu \nu}{ }^{C} F_{\rho \sigma}{ }^{0}+(t+\bar{t})^{A}(t+\bar{t})^{B}(t+\bar{t})^{C} F_{\mu \nu}{ }^{0} F_{\rho \sigma}{ }^{0}\right]
\end{gathered}
$$

and finally the last term is rewritten as,

$$
\begin{aligned}
- & E C_{A B C} \sigma^{A} \sigma^{B} \sigma^{C}\left[\frac{1}{8} R-4 D-\frac{39}{2} T^{A B} T_{A B}\right]=-\frac{1}{2} \mathrm{ie} C_{A B C}(t-\bar{t})^{A}(t-\bar{t})^{B}(t-\bar{t})^{C} \\
\times & {\left[\left(\frac{1}{6} R-D\right)\left|X^{0}\right|^{2}-\frac{1}{16} Y_{i j}{ }^{0} Y^{i j 0}-\left|\mathcal{D}_{\mu} X^{0}\right|^{2}+\frac{3}{2}\left(\mathcal{D}_{\mu}\left|X^{0}\right|\right)^{2}+\frac{1}{32} F_{a b}{ }^{0} F^{a b 0}\right.} \\
& \left.+\frac{1}{32}\left[\left(\varepsilon_{i j} T_{a b}{ }^{i j} \bar{X}^{0}-F_{a b}^{-0}\right)^{2}+\text { h.c. }\right]\right] .
\end{aligned}
$$


The resulting Lagrangian can be expressed in terms of the following homogeneous and holomorphic function of degree two [16],

$$
F(X)=-\frac{1}{2} \frac{C_{A B C} X^{A} X^{B} X^{C}}{X^{0}},
$$

which encodes the bosonic terms of the Lagrangian according to

$$
\begin{aligned}
e^{-1} \mathcal{L}_{\text {bosonic }}= & -\mathrm{i}\left(\mathcal{D}_{\mu} X^{I} \mathcal{D}^{\mu} \bar{F}_{I}-\mathcal{D}_{\mu} \bar{X}^{I} \mathcal{D}^{\mu} F^{I}\right)+\mathrm{i}\left(X^{I} \bar{F}_{I}-\bar{X}^{I} F_{I}\right)\left(\frac{1}{6} R-D\right) \\
& +\frac{1}{4} \mathrm{i}\left[F_{I J} F_{\mu \nu}^{-I} F^{-\mu \nu J}-\bar{F}_{I J} F_{\mu \nu}^{+I} F^{+\mu \nu J}\right] \\
& +\left[\frac{1}{8} \bar{X}^{I} N_{I J} F^{-a b I} T_{a b}{ }^{i j} \varepsilon_{i j}-\frac{1}{64} \bar{X}^{I} N_{I J} \bar{X}^{J}\left(T_{\mu \nu}{ }^{i j} \varepsilon_{i j}\right)^{2}+\text { h.c. }\right] \\
& +N_{I J} Y_{i j}{ }^{I} Y^{i j J} .
\end{aligned}
$$

where $N_{I J}=-\mathrm{i} F_{I J}+\mathrm{i} \bar{F}_{I J}$

Finally, we turn to the reduction of the four-derivative coupling involving the vector multiplets and the Weyl multiplet, first introduced in [6]. Here, we refrain from giving full details of the invariants in both five and four dimensions, concentrating on the identification of the relevant functions arising under dimensional reduction. We use the conventions of [13] and concentrate on the following terms, ${ }^{3}$

$$
\begin{aligned}
8 \pi^{2} \mathcal{L}_{\mathrm{vww}}= & \frac{1}{4} E c_{A} Y_{i j}{ }^{A} T^{C D} R_{C D k}{ }^{j}(V) \varepsilon^{k i} \\
& +E c_{A} \sigma^{A}\left[\frac{1}{64} R_{C D}{ }^{E F}(M) R_{E F}{ }^{C D}(M)+\frac{1}{96} R_{M N j}{ }^{i}(V) R^{M N}{ }_{i}^{j}(V)\right] \\
& -\frac{1}{128} \mathrm{i} \varepsilon^{M N P Q R} c_{A} W_{M}{ }^{A}\left[R_{N P}{ }^{C D}(M) R_{Q R C D}(M)+\frac{1}{3} R_{N P j}{ }^{i}(V) R_{Q R i}{ }^{j}(V)\right] \\
& +\frac{3}{16} E c_{A}\left(10 \sigma^{A} T_{B C}-F_{B C}{ }^{A}\right) R(M)_{D E}{ }^{B C} T^{D E}+\cdots,
\end{aligned}
$$

where $R(M)_{M N}{ }^{C D}$ coincides with the $5 D$ Weyl tensor, up to certain additions implied by supersymmetry. Upon reduction of (4.16) to four dimensions one obtains a $4 D$ supersymmetric Lagrangian with higher-derivative couplings. For our purpose, it suffices to concentrate on the terms that involve the tensors $R(M)_{a b}{ }^{c d}$ and/or $R(\mathcal{V})_{a b}{ }^{i}{ }_{j}$. As it turns out these terms can be decomposed into three sets that exhibit a mutually different structure. Subsequently we will try and identify these sets in terms of independent $4 D$ supersymmetric Lagrangians.

The first set of terms is given by,

$$
8 \pi^{2} \mathcal{L}_{\mathrm{vww}} \rightarrow-\frac{1}{64} \mathrm{i} c_{A} t^{A}\left[2 R(M)_{a b}^{-c d} R(M)_{c d}^{-a b}+R(\mathcal{V})_{a b j}^{-i} R(\mathcal{V})^{-a b j}{ }_{i}\right]
$$

\footnotetext{
${ }^{3}$ Here we use the index $A$ to label the $5 D$ vector multiplets and the indices $B, C, \ldots$ to indicate $5 D$ tangent-space indices.
} 


$$
\begin{aligned}
& -\frac{1}{512} \mathrm{i} \varepsilon_{m n} T^{a b m n}\left(X^{0}\right)^{-1} c_{A}\left(Y^{i j A}-t^{A} Y^{i j 0}\right) R(\mathcal{V})_{a b j}^{-k} \varepsilon_{k i} \\
& +\frac{1}{256} \mathrm{i} c_{A}\left(X^{0}\right)^{-1} \varepsilon_{i j} T^{c d i j} R(M)^{a b}{ }_{c d}\left(F_{a b}^{-A}-t^{A} F_{a b}^{-0}\right) \\
& + \text { h.c. }
\end{aligned}
$$

which, as we shall see, belongs to a $4 D$ supersymmetric invariant based on a chiral superspace integral $[7,8]$. Here $R(M)_{a b}{ }^{c d}$ denotes the $4 D$ Weyl tensor.

The second set of terms involves expressions that cannot be readily associated with a known $N=2$ supersymmetric invariant,

$$
\begin{aligned}
8 \pi^{2} \mathcal{L}_{\mathrm{vww}} \rightarrow & -\frac{1}{384} \mathrm{i} c_{A} t^{A}\left[\frac{2}{3} \mathcal{R}_{a b} \mathcal{R}^{a b}+R(\mathcal{V})_{a b j}^{+i} R(\mathcal{V})^{+a b j}{ }_{i}\right] \\
& -\frac{1}{768} \mathrm{i} c_{A}\left(t^{A}-\bar{t}^{A}\right)\left(X^{0}\right)^{-1} \varepsilon_{i j} T^{c d i j} R(M)^{a b}{ }_{c d} F_{a b}^{-0} \\
& + \text { h.c. }
\end{aligned}
$$

A conspicuous feature of this term is its dependence on the Ricci tensor $\mathcal{R}_{a b}$. It is rather obvious that this term is not related to a chiral superspace invariant. The same comment applies to the third set of terms, given by,

$$
\begin{aligned}
8 \pi^{2} \mathcal{L}_{\mathrm{vww}} \rightarrow & \frac{1}{384} \mathrm{i} c_{A} R(\mathcal{V})^{+a b k}{ }_{j} \varepsilon_{k i}\left|X^{0}\right|^{-2}\left[F_{a b}^{+A} Y^{i j 0}-F_{a b}^{+0} Y^{i j A}+(t-\bar{t})^{A} F_{a b}^{+0} Y^{i j 0}\right] \\
& +\frac{1}{1536} \mathrm{i} \varepsilon^{m n} T^{a b}{ }_{m n}\left(\bar{X}^{0}\right)^{-1} c_{A}\left(Y^{i j A}-\left(2 t^{A}-\bar{t}^{A}\right) Y^{i j 0}\right) R(\mathcal{V})_{a b}^{+k}{ }_{j} \varepsilon_{k i} \\
& + \text { h.c. }
\end{aligned}
$$

We have now completely determined the terms that depend on $R(\mathcal{V})_{a b}{ }^{i}{ }_{j}$, as well as the terms in (4.16) that depend explicitly on $R(M)_{a b}{ }^{c d}$. However, (4.16) also contains a term with a double derivative proportional to $T^{A B} D^{C} D_{A} T_{B C}$ which can in principle give rise to additional curvature terms upon reordering derivatives combined with partial integrations. The evaluation of some of these terms remains therefore a little ambiguous at this stage, also because the final result may be subject to the similar rearrangements. Nevertheless the results determined above are sufficient to discuss the structure of the resulting $4 D$ Lagrangians.

As was mentioned above, the terms (4.17) exhibit an underlying holomorphic structure that is characteristic for an invariant based on a chiral superspace integral (sometimes referred to as an 'F-term'). Such an invariant is well known and it can again be encoded into a holomorphic function. This function can be included into the function (4.14) by introducing a dependence on an extra complex field, $\hat{A}$, which is equal to $\hat{A}=\left(T_{a b}{ }^{i j} \varepsilon_{i j}\right)^{2}$. In the case at hand, the dependence on $\hat{A}$ is linear, but for a general $4 D$ Lagrangian the function has to be holomorphic and homogeneous of second degree [7, 8]. As it turns out, the modified function $F(X, \hat{A})$ that correctly encodes the sum of (4.15) and (4.17), equals,

$$
F(X, \hat{A})=-\frac{1}{2} \frac{C_{A B C} X^{A} X^{B} X^{C}}{X^{0}}-\frac{1}{2048} \frac{c_{A} X^{A}}{X^{0}} \hat{A}
$$


where the higher-order derivative Lagrangian encoded in this function reads as [8],

$$
\begin{aligned}
e^{-1} \mathcal{L}= & -4 \mathrm{i} F_{\hat{A} I} T^{c d l m} \varepsilon_{l m}\left[2 R(M)_{c d}^{a b}\left(F_{a b}^{-I}-\frac{1}{4} \bar{X}^{I} T_{a b}{ }^{i j} \varepsilon_{i j}\right)-\varepsilon_{k i} R(\mathcal{V})_{c d}{ }_{j}{ }_{j} Y^{i j I}\right] \\
& +16 \mathrm{i} F_{\hat{A}}\left[2 R(M)^{-c d}{ }_{a b} R(M)_{a b}^{-c d}+R(\mathcal{V})^{-a b k}{ }_{l} R(\mathcal{V})_{a b k}^{-l}\right]+\cdots \\
& + \text { h.c. }
\end{aligned}
$$

Here we only give the terms relevant for the comparison with (4.17).

The interpretation of (4.18) is, however, less clear, as terms of this type have never been written down explicitly in $N=2$ supergravity. Supersymmetric invariants that contain the square of the Ricci tensor have been written down in $N=1$ supergravity, often in the context of a supersymmetrization of the Gauss-Bonnet term [17-20]. The latter is a topological invariant whose integral is proportional to the Euler characteristic of the corresponding manifold. The emergence of this new supersymmetric coupling in the reduction from (4.16) constitutes a new result. A brief perusal of the various terms arising in this reduction shows that it will involve quite a variety of new couplings. It is obviously of importance to understand the structure of this invariant and its possible implications for $4 D$ black hole entropy, also in view of the recent discussion in [21].

Finally we come to the invariant that contains the terms (4.19). On closer inspection it turns out the the dimensional reduction of (4.16) involves also terms quartic in the field strengths. These couplings belong to the class of invariants constructed in [9]. One of these invariants is indeed quartic in the field strengths and it contains the following characteristic terms,

$$
\begin{aligned}
& e^{-1} \mathcal{L}= \frac{1}{4} \mathcal{H}_{I J \bar{K} \bar{L}}\left(F_{a b}^{-I} F^{-a b J}-\frac{1}{2} Y_{i j}{ }^{I} Y^{i j J}\right)\left(F_{a b}^{+K} F^{+a b L}-\frac{1}{2} Y^{i j K} Y_{i j}{ }^{L}\right) \\
&-\left\{\mathcal{H}_{I J \bar{K}}\left(F^{-a b I} F_{a b}^{-J}-\frac{1}{2} Y_{i j}^{I} Y^{J i j}\right)\left(\square_{\mathrm{c}} X^{K}+\frac{1}{8} F_{a b}^{-K} T^{a b k l} \varepsilon_{k l}\right)+\text { h.c. }\right\} \\
&+ \mathcal{H}_{I \bar{J}}\left[4\left(\square_{\mathrm{c}} \bar{X}^{I}+\frac{1}{8} F_{a b}^{+I} T^{a b}{ }_{i j} \varepsilon^{i j}\right)\left(\square_{\mathrm{c}} X^{J}+\frac{1}{8} F_{a b}^{-J} T^{a b i j} \varepsilon_{i j}\right)\right. \\
&+8 \mathcal{D}_{a} F^{-a b I} \mathcal{D}_{c} F^{+c}{ }_{b}{ }^{J}-\mathcal{D}_{a} Y_{i j}{ }^{I} \mathcal{D}^{a} Y^{i j J} \\
&+8 \mathcal{R}^{\mu \nu} \mathcal{D}_{\mu} X^{I} \mathcal{D}_{\nu} \bar{X}^{J} \\
&-\left[\varepsilon^{i k} Y_{i j}{ }^{I}\left(F^{+a b J}-\frac{1}{4} X^{J} T^{a b}{ }_{l m} \varepsilon^{l m}\right) R(\mathcal{V})_{a b}{ }^{j} k\right. \\
&
\end{aligned}
$$

where $\mathcal{H}(X, \bar{X})$ is a real homogeneous function of degree zero. These invariants, often called 'D-terms', are based on a full superspace integral and they are obviously not encoded in terms of holomorphic functions. In this particular case the invariant depends only on the mixed multiple derivatives of $\mathcal{H}(X, \bar{X})$, so that one is dealing with an underlying 
Kähler equivalence,

$$
\mathcal{H}(X, \bar{X}) \rightarrow \mathcal{H}(X, \bar{X})+\Lambda(X)+\bar{\Lambda}(\bar{X}) .
$$

which is based on the fact that a chiral superfield vanishes when integrated over the full superspace. Obviously the mixed derivative $\mathcal{H}_{I \bar{J}}$ can be regarded as a Kähler metric.

It is now straightforward to show that (4.22) generates the terms in (4.19) provided that,

$$
\begin{aligned}
& \mathcal{H}_{0 \bar{A}}=-\frac{1}{384} \mathrm{i} c_{A}\left|X^{0}\right|^{-2}, \\
& \mathcal{H}_{0 \overline{0}}=-\frac{1}{384} \mathrm{i} c_{A}\left(t^{A}-\bar{t}^{A}\right)\left|X^{0}\right|^{-2},
\end{aligned}
$$

so that (up to a Kähler transformation),

$$
\mathcal{H}(X, \bar{X})=\frac{1}{384} \mathrm{i} c_{A}\left(t^{A} \ln \bar{X}^{0}-\bar{t}^{A} \ln X^{0}\right) .
$$

\section{The $4 \mathrm{D} / 5 \mathrm{D}$ connection and the BPS spinning black hole}

In this section we return to some open questions that arose in the calculation of the entropy of spinning BPS black holes based on $5 D$ supersymmetric Lagrangians with higherderivative couplings. Since the entropy must be expressed in terms of the charges and the angular momentum of the black hole, these quantities will have to be determined as well. The first calculations were carried out in $[10,11]$, where both the near-horizon attractor geometry and the field equations leading to the global solution were studied. The main results were that the reduced $5 D$ field equations were inconsistent with the known $4 D$ equations, and that the $5 D$ and $4 D$ electric charges differ by a constant shift induced by the higher-order derivative couplings. Another study was undertaken in [13]. It was aimed at demonstrating that all the information on charges, angular momentum and entropy can be obtained from the near-horizon data, and at providing an independent verification of the results of $[10,11]$. As it turns out the results of the two studies did not entirely agree. The precise results for the electric charges were different in the case of non-zero angular momentum, and furthermore the expressions for the angular momentum were different. This could have been interpreted as evidence that the relevant data cannot be determined from the near-horizon analysis alone. However, this seems unlikely in view of the fact that the same study in [13] did lead to a full determination of the entropy, electric charges and angular momenta for BPS black rings, confirming many independent results based on field theoretic solutions and on microstate counting [22-27].

In both of these studies the results were compared to the corresponding results for four-dimensional black holes, although it is questionable whether the results should a priori be the same after straightforward dimensional reduction. Indeed, for black rings it was noted there are subtle differences between the four- and five-dimensional charges, and the 
electric charges are not additively conserved in five dimensions as a result of the ChernSimons terms. It seems likely that the differences between results obtained from theories that are related by dimensional reduction originate from topological subtleties related to Chern-Simons terms, which do not carry over to the lower dimension. Calculations of supergravity solutions are notoriously difficult in the presence of higher-derivative interactions, but the results of the present paper will enable us to confirm once more that this expectation is indeed correct, as we will demonstrate below in a relatively simple model. Subsequently we will discuss the topological features related with the Chern-Simons contributions in more detail.

To examine how the results of these calculations based on the $5 D$ supergravity relate to those based on the $4 D$ supergravity, we consider a simple model action, ${ }^{4}$

$$
8 \pi^{2} S=\int \mathrm{d}^{5} x\left\{-E\left(\frac{1}{2} R+\frac{1}{4} F_{M N}{ }^{2}\right)+\frac{\zeta}{128} \varepsilon^{M N P Q R} W_{M} R_{N P}{ }^{A B} R_{Q R A B}\right\},
$$

where $\zeta$ is the strength of the higher-derivative mixed gauge-gravitational Chern-Simons term and $F_{M N}=2 \partial_{[M} W_{N]}$.

Let us assume that this theory has an extremal black hole solution, whose near-horizon geometry is a fibration of $\mathrm{AdS}_{2} \times S^{2}$,

$$
\begin{aligned}
\mathrm{d} s^{2} & =v_{1}\left(-r^{2} \mathrm{~d} t^{2}+r^{-2} \mathrm{~d} r^{2}\right)+v_{2}\left(\mathrm{~d} \theta^{2}+\sin ^{2} \theta \mathrm{d} \varphi^{2}\right)+\phi^{-2}(\mathrm{~d} \psi+B)^{2}, \\
B & =e^{0} r \mathrm{~d} t+p^{0} \cos \theta \mathrm{d} \varphi, \\
W & =W^{4}+\chi(\mathrm{d} \psi+B), \\
W^{4} & =e r \mathrm{~d} t+p \cos \theta \mathrm{d} \varphi,
\end{aligned}
$$

Note that $B$ specifies a value for the Kaluza-Klein gauge field. The gauge field $W_{M}$, decomposed according to the standard Kaluza-Klein ansatz with its fifth component denoted by $\chi$, leads to the field strengths,

$$
F_{t r}=-\left(e+\chi e^{0}\right), \quad F_{\theta \varphi}=-\left(p+\chi p^{0}\right) \sin \theta .
$$

In what follows we further restrict the background by choosing,

$$
p=0, \quad v_{1}=v_{2}=v^{2}, \quad\left(e^{0}\right)^{2}+\left(p^{0}\right)^{2}=v^{2} \phi^{2} .
$$

In that case we are more in line with the BPS near-horizon horizon geometry in the full $5 D$ supergravity used in [13], and furthermore the gauge field $W_{M}$ will be globally defined, which is important for what follows. With these assumptions the line element can then be written as,

$$
\begin{aligned}
d s^{2}= & -\phi^{-2} \rho^{4}\left(p^{0} \mathrm{~d} t-\frac{e^{0}}{\rho^{2}}\left(\cos \theta \mathrm{d} \varphi+\frac{1}{p^{0}} \mathrm{~d} \psi\right)\right)^{2} \\
& +\frac{4 v^{2}}{\rho^{2}}\left(\mathrm{~d} \rho^{2}+\frac{\rho^{2}}{4}\left(\mathrm{~d} \theta^{2}+\mathrm{d} \varphi^{2}+\frac{1}{\left(p^{0}\right)^{2}} \mathrm{~d} \psi^{2}+\frac{2}{p^{0}} \cos \theta \mathrm{d} \varphi \mathrm{d} \psi\right)\right)
\end{aligned}
$$

\footnotetext{
${ }^{4}$ Unlike in the other sections we do not use Pauli-Källén conventions, but conventions with signature $(-,+,+,+,+)$ and $\varepsilon^{01234}=-1$. This leaves our final results unchanged.
} 
where we used the definition $\rho=\sqrt{r}$. To make $p^{0}$ unambiguous we fix the periodicity interval for $\psi$ to $4 \pi$. The second term of the line element then corresponds to a flat metric, up to an overall warp factor $4 v^{2} \rho^{-2}$. Clearly for $\left|p^{0}\right|=1$ we cover the whole fourdimensional space $\mathbb{R}^{4}$. For $\left|p^{0}\right| \neq 1$ we have a conical singularity at the origin. In all cases the three-dimensional horizon is located at $r=0$ and its cross-sectional area is equal to

$$
A_{3}=\int_{\Sigma_{\mathrm{hor}}}=16 \pi^{2} v^{2} \phi^{-1}
$$

The bi-normal $\varepsilon_{M N}$ that characterizes the null surface at the horizon equals $\varepsilon_{t r}=-\varepsilon_{r t}=$ $v^{2}$.

Subsequently we determine the electric charge associated with the gauge field $W_{M}$,

$$
\begin{aligned}
q^{(5)} & =-4\left(e+\chi e^{0}\right) \phi^{-1}-\frac{\zeta}{128 \pi^{2}} \mathcal{Q}_{\mathrm{CS}} \\
& =-4\left(e+\chi e^{0}\right) \phi^{-1}-\frac{3 \zeta}{16} \frac{p^{0}\left(e^{0}\right)^{2}}{\left[\left(p^{0}\right)^{2}+\left(e^{0}\right)^{2}\right]^{2}},
\end{aligned}
$$

where we used the expression for the integrated Chern-Simons term on the horizon,

$$
\begin{aligned}
\mathcal{Q}_{\mathrm{CS}} & =\int_{\Sigma_{\text {hor }}} \mathrm{d} \theta \mathrm{d} \varphi \mathrm{d} \psi \frac{\varepsilon_{M N}}{2 v^{2}} \varepsilon^{M N P Q R} \Gamma_{P S}{ }^{T}\left(\partial_{Q} \Gamma_{R T}{ }^{S}-\frac{2}{3} \Gamma_{Q T}{ }^{U} \Gamma_{R U} S\right) \\
& =24 \pi^{2} \frac{p^{0}\left(e^{0}\right)^{2}}{\left[\left(p^{0}\right)^{2}+\left(e^{0}\right)^{2}\right]^{2}} .
\end{aligned}
$$

In what follows this last result will play a crucial role, because the Chern-Simons term does not transform as a density under diffeomorphisms. This implies that one may obtain a different answer upon writing the metric in different coordinates. To ensure that the coordinate singularity at $r=0$ is not causing complications, we also evaluated the horizon area (5.6) and the Chern-Simons charge (5.8) in a regular near-horizon metric by converting to new coordinates, which gave rise to identical results. Nevertheless, we have also found examples of different metrics which did indeed give rise to different results for the integrated Chern-Simons term. For the moment we will proceed, assuming that (5.8) represents the correct result. At the end of the section we will reconsider this issue from a topological perspective, which will lend further support to the correctness of the above result.

We also determine the angular momentum, which, according to (5.2), will vanish when $e^{0}=\chi=0$. One first evaluates the Noether potential for diffeomorphisms parametrized in terms of a vector $\xi^{M}$ (the relevant formulae can be obtained from [13]),

$$
\begin{aligned}
8 \pi^{2} \mathcal{Q}^{M N}(\xi)=\nabla^{[M} \xi^{N]}+ & F^{M N} W_{P} \xi^{P}-\frac{\zeta}{64} E^{-1}\left[2 \varepsilon^{M N P Q R} W_{P} R_{Q R}{ }^{S T} \nabla_{S} \xi_{T}\right. \\
& \left.-2 \varepsilon^{P Q R S[M} F_{P Q} R_{R S}^{N] T} \xi_{T}+\varepsilon^{P Q R S T} F_{P Q} R_{R S}^{M N} \xi_{T}\right],
\end{aligned}
$$

where the Riemann tensor can be written as,

$$
\begin{aligned}
\mathcal{R}_{M N}{ }^{P Q}=-\frac{1}{2} \phi^{-2} & \left(t_{M N} t^{P Q}+t_{M}^{[P} t_{N}^{Q]}\right. \\
& \left.+2 \delta_{[M}^{[P} t_{N] R} t^{Q] R}-\frac{1}{2} \delta_{[M}^{P} \delta_{N]}^{Q} t_{R T} t^{R T}\right),
\end{aligned}
$$


with the non-vanishing components of the anti-symmetric tensor $t_{M N}$ given by

$$
t_{t r}=p^{0}, \quad t_{\theta \varphi}=-e^{0} \sin \theta .
$$

Subsequently one considers the periodic Killing vector $\partial / \partial \psi$ associated with rotations. In this case we have $\xi_{M} \mathrm{~d} x^{M}=\phi^{-2}\left(\mathrm{~d} \psi+e^{0} r \mathrm{~d} t+p^{0} \cos \theta \mathrm{d} \varphi\right)$, so that the nonvanishing derivatives of $\xi_{M}$ equal,

$$
\nabla_{[t} \xi_{r]}=-\frac{1}{2} e^{0} \phi^{-2}, \quad \nabla_{[\theta} \xi_{\varphi]}=-\frac{1}{2} p^{0} \phi^{-2} .
$$

Substituting the above results into (5.9) and integrating over the horizon leads to the following expression for the angular momentum,

$$
\begin{aligned}
J_{\psi} & \equiv \int_{\Sigma_{\mathrm{hor}}} \varepsilon_{M N} \mathcal{Q}^{M N}\left(\xi^{\psi}\right) \\
& =-4 \phi^{-1}\left[\chi e+\left(\chi^{2}+\frac{1}{2} \phi^{-2}\right) e^{0}\right]-\frac{\zeta}{16} \frac{p^{0}\left[2 e e^{0}+3 \chi\left(3\left(e^{0}\right)^{2}-\left(p^{0}\right)^{2}\right)\right]}{\left[\left(e^{0}\right)^{2}+\left(p^{0}\right)^{2}\right]^{2}} .
\end{aligned}
$$

We would like to briefly compare these results to the results based on the $4 D$ action that one obtains upon dimensional reduction of the $5 D$ action (5.1). The corresponding Lagrangian reads,

$$
\begin{aligned}
2 \pi \mathcal{L}=- & \sqrt{|g|} \phi^{-1}\left\{\frac{1}{2} R+\frac{1}{8}\left(\phi^{-2}+2 \chi^{2}\right) F_{\mu \nu}^{0}{ }^{2}+\frac{1}{4} F_{\mu \nu}{ }^{2}+\frac{1}{2} \chi F_{\mu \nu}^{0} F^{\mu \nu}\right\} \\
+ & \frac{1}{128} \zeta \varepsilon^{\mu \nu \rho \sigma}\left\{\chi R_{\mu \nu}^{\lambda \tau} R_{\rho \sigma \lambda \tau}+\chi \phi^{-2} R_{\mu \nu}{ }^{\lambda \tau}\left[F_{\rho \lambda}^{0} F_{\sigma \tau}^{0}+F_{\rho \sigma}^{0} F_{\lambda \tau}^{0}\right]\right. \\
& +\frac{1}{4} \chi \phi^{-4} F_{\mu \nu}^{0}\left[2 F_{\rho \lambda}^{0} F_{\sigma \tau}^{0} F^{0 \lambda \tau}+F_{\rho \sigma}^{0} F_{\lambda \tau}^{0}\right]+\frac{1}{2} \chi \phi^{-2} \mathcal{D}_{\lambda} F_{\mu \nu}^{0} \mathcal{D}^{\lambda} F_{\rho \sigma}^{0} \\
& \left.+F_{\mu \nu}\left[\phi^{-2} R_{\rho \sigma}{ }^{\lambda \tau} F_{\lambda \tau}^{0}+\frac{1}{4} \phi^{-4}\left[F_{\lambda \tau}^{0}{ }^{2} F_{\rho \sigma}^{0}+2 F^{0 \lambda \tau} F_{\rho \lambda}^{0} F_{\sigma \tau}^{0}\right]\right]\right\},
\end{aligned}
$$

where $F_{\mu \nu}^{0}=2 \partial_{[\mu} B_{\nu]}$. Eventually we assume constant values for $v, \phi$ and $\chi$, and therefore we have suppressed above the contributions from space-time derivatives of these fields. Furthermore, we have absorbed an overall factor of $4 \pi$ to account for the length of the interval of the extra $5 D$ coordinate $\psi$.

The $4 D$ line element follows from (5.5),

$$
\mathrm{d} s^{2}=v^{2}\left(-r^{2} \mathrm{~d} t^{2}+r^{-2} \mathrm{~d} r^{2}+\mathrm{d} \theta^{2}+\sin ^{2} \theta \mathrm{d} \varphi^{2}\right) .
$$

Therefore the near-horizon geometry equals $\mathrm{AdS}_{2} \times S^{2}$ and the Riemann tensor decomposes into the Riemann tensors associated with each of the two maximally symmetric factors. In addition we have the field strengths,

$$
F_{t r}^{0}=-e^{0}, \quad F_{\theta \varphi}^{0}=-p^{0} \sin \theta, \quad F_{t r}=-e, \quad F_{\theta \varphi}=0 .
$$


Making use of this fact we determine the value of the $4 D$ electric charges associated with the $4 D$ gauge fields $B_{\mu}$ and $W_{\mu}$,

$$
\begin{aligned}
q^{(4)} & =-4 \phi^{-1}\left(e+\chi e^{0}\right)-\frac{\zeta}{16} \frac{p^{0}\left(5\left(e^{0}\right)^{2}+2\left(p^{0}\right)^{2}\right)}{\left[\left(e^{0}\right)^{2}+\left(p^{0}\right)^{2}\right]^{2}} \\
q_{0} & =-4 \phi^{-1}\left[\chi e+\left(\chi^{2}+\frac{1}{2} \phi^{-2}\right) e^{0}\right]-\frac{\zeta}{16} \frac{p^{0}\left[2 e e^{0}+3 \chi\left(3\left(e^{0}\right)^{2}-\left(p^{0}\right)^{2}\right)\right]}{\left[\left(e^{0}\right)^{2}+\left(p^{0}\right)^{2}\right]^{2}}
\end{aligned}
$$

Comparing these charges to the results for the five-dimensional charge and angular momentum, specified by (5.7) and (5.13), respectively, we find,

$$
\begin{aligned}
q^{(4)} & =q^{(5)}-\frac{\zeta}{8} \frac{p^{0}}{\left(e^{0}\right)^{2}+\left(p^{0}\right)^{2}} \\
q_{0} & =J_{\psi} .
\end{aligned}
$$

The value of the four-dimensional charge $q_{0}$, associated with the Kaluza-Klein gauge field, coincides exactly with the five-dimensional angular momentum of the spinning black hole. On the other hand, the five-dimensional charge associated with the vector multiplet, $q^{(5)}$, differs from the four-dimensional charge $q^{(4)}$ which is obtained after the straightforward reduction of the Lagrangian. And furthermore this difference is directly related to the Chern-Simons term. These conclusions are consistent with the results derived in [13]. In [11] it was also found that the five- and four-dimensional charges are not the same and are related by a shift. However, as it turns out, the latter shift is different from the one above, because it does not depend on $e^{0}$.

In the remainder of this section we explain how this last phenomenon can be understood in terms of the topology associated with the Chern-Simons term. The latter arises from the defining condition,

$$
\mathrm{d} \operatorname{Tr}\left[C \wedge \mathrm{d} C-\frac{2}{3} C \wedge C \wedge C\right]=\frac{1}{4} \operatorname{Tr}[R \wedge R],
$$

where $C$ is an appropriate matrix-valued connection equal to the Christoffel or to the spin connection. If there is a non-trivial flux of $R \wedge R$ along a 4-cycle, it is not possible to define the Chern-Simons term globally, but only on patches connected by an appropriate closed, non-exact, gauge transformation. Here we may regard the Chern-Simons term as a composite 3 -form potential constructed from the metric, rather than as a fundamental gauge field. The gauge transformation between the patches is thus induced by certain diffeomorphisms, where we assume that any additional ambiguity associated with the underlying diffeomorphisms will have no cohomological consequences.

In four dimensions, the flux of $R \wedge R$ is a topological invariant, so that the ChernSimons term cannot be defined as a gauge potential, as it would carry no degrees of freedom. However, when the Chern-Simons term is viewed as a 3 -form gauge potential in five space-time dimensions, one finds that its magnetic dual is a scalar field. The situation is 
thus analogous to that of a magnetic monopole located at some point in a three-dimensional Euclidean space, except that here we are dealing with a point in a five-dimensional spacetime. Likewise, from that point in space-time there will be a Dirac string emanating from it, extending to infinity. Obviously this extension to infinity has to be compatible with the fact that we are dealing with stationary solutions. We recall that the choice for the string is associated with a certain regular gauge patch and the transition functions between different patches are provided by gauge transformations (in this case induced by diffeomorphisms). The strings associated with two different patches are connected by a corresponding twodimensional surface which encodes the corresponding gauge transformation.

We are interested in computing the integral of the Chern-Simons term in (5.19) over a spatial 3-dimensional surface, as a contribution to the electric charge. In the analogous situation of the monopole in three spatial dimensions, this integral is a Wilson line whose value may differ on each patch depending on whether it encircles the Dirac string or not. The difference is given by the gauge transformation between the patches and is proportional to the flux of the corresponding field strength given by $R \wedge R$.

Without loss of generality, we may employ two patches and impose that the corresponding Dirac strings are timelike and intersect at most once with each spatial slice. ${ }^{5}$ The question is then how precisely the string will extend through space-time. In the context of a black hole background, there are only two acceptable choices, namely, that the string will move to spatial infinity at large time, or that the string will remain behind the black hole horizon, so that the unphysical string singularity is not observable.

It follows that the surface connecting the strings associated with two different sections define a semi-infinite plane along the time and radial directions. The closed but non-exact gauge transformation, $\beta^{0}$, which connects the two patches, is given by the normal form $\beta^{0}$ of this plane [28-30]. For the metric above, this reads

$$
\beta^{0}=\frac{p^{0}}{\left(e^{0}\right)^{2}+\left(p^{0}\right)^{2}} \sin \theta \mathrm{d} \theta \wedge \mathrm{d} \varphi \wedge \mathrm{d} \psi .
$$

Here, the factor $p^{0}$ is implied by the induced metric on the $S^{3}$ defined by constant values of $t$ and $r$, and the overall normalisation is fixed by demanding that in the static limit the integral over $\beta^{0}$ is equal to $2 / p^{0}$, i.e. equal to the flux of $\operatorname{Tr}[R \wedge R]$ for a Gibbons-Hawking base space. Therefore, the value of the Chern-Simons term in the patch that contains the Dirac string singularity, is related to the value in the regular patch, where the singularity is located at infinity, by

$$
\left.\operatorname{Tr}\left[C_{[\mu} \partial_{\nu} C_{\rho]}-\frac{2}{3} C_{[\mu} C_{\nu} C_{\rho]}\right]\right|_{\text {sing }}=\left.\operatorname{Tr}\left[C_{[\mu} \partial_{\nu} C_{\rho]}-\frac{2}{3} C_{[\mu} C_{\nu} C_{\rho]}\right]\right|_{\text {reg }}+\beta_{\mu \nu \rho}^{0} .
$$

The gauge transformation in (5.21) changes the position of the intersection of the Dirac string within a given spatial slice. We conclude that the integral of the above Chern-Simons

\footnotetext{
${ }^{5}$ This condition can be relaxed by introducing more than two patches.
} 
term over a 3-surface can take two distinct values depending whether the intersection point is contained or not in that 3-surface. Therefore, the electric charges of BPS spinning black holes in a five-dimensional theory containing a mixed gauge/gravitational Chern-Simons term can also take two different values, depending on how the patches are chosen.

In an asymptotically flat five-dimensional setting one can impose regularity in the bulk of the solution, pushing the Dirac brane to infinity. The connection used to evaluate the integrated Chern-Simons term in (5.8) in consistent with this requirement, in line with the general view that a non-trivial Taub-NUT charge is not considered to be part of the black hole in the center, so that no singularity associated to $R \wedge R$ should appear. This parallels the approach used in the microscopic counting, where the large-charge limit is taken for the electric charges but not for the Taub-NUT charge.

One can now also consider the corresponding four-dimensional solution based on (5.14). In that setting, it is not acceptable to have a gauge-dependent singularity present near spatial infinity. However, a physical solution can still be obtained if the singularity is hidden behind the horizon, which amounts to a change of patch as in (5.21). From a four-dimensional perspective, this corresponds to the addition of a delta source singularity interpreted as a magnetic monopole associated with the Kaluza-Klein gauge field.

The results above are in agreement with the results from the near-horizon analysis for spinning five-dimensional BPS black holes given in [13], where it was found that the difference between the four- and five-dimensional charges differ by a shift that depends both on $p^{0}$ and on $e^{0}$. In that case the angular momentum is proportional to $e^{0}$, so that the shift will depend on the angular momentum. This result differs from that in [11], in spite of the fact that there the singularity associated with the Taub-NUT charge has also been moved to spatial infinity. However, for reasons that are not clear to us, the coefficient of that singularity depended only on $p^{0}$, so that the difference between the four- and fivedimensional electric charges was constant and did not depend on the angular momentum.

\section{Concluding remarks}

In this paper we studied the off-shell dimensional reduction of five-dimensional $N=1$ conformal supergravity to four space-time dimensions. We obtained the full dictionary expressing the $5 D$ fields in terms of the $4 D$ fields and showed in some detail how to connect to the standard $N=2$ superconformal Lagrangians in four dimensions. The advantage of performing the reduction off-shell is that it allows one to make a precise comparison of the two theories beyond the usual two-derivative actions, by connecting the four-derivative invariants on both sides.

Somewhat unexpectedly, we found that upon reduction of the four-derivative supersymmetric action in five dimensions, which contains terms quadratic in the Riemann tensor, one finds terms that are not compatible with the two four-derivative $N=2$ supersymmetric invariants known in four dimensions. In this way we deduce the presence of at least 
one new four-dimensional invariant that is quadratic in the Ricci tensor, whose complete structure remains to be uncovered. Terms like these will be required when considering the $N=2$ supersymmetric extension of the Gauss-Bonnet invariant.

As a further application of our reduction scheme, we studied the effect of the mixed gauge/gravitational Chern-Simons term, contained in the five-dimensional four-derivative action, on the definition of the electric charge for spinning supersymmetric black holes. Consistent with previous results on five-dimensional BPS black holes [13], we find a shift in the charges upon dimensional reduction to four dimensions, whose value depends on the angular momentum. Similar examples of this phenomenon have been discovered at the two-derivative level for black rings. Just as in that case, the subtleties can be understood from the presence of Dirac branes and the associated sections.

The off-shell approach to dimensional reduction developed in this paper is not specific to five dimensions and can be applied to other situations. One interesting example would be the reduction from four to three dimensions, especially in connection to the c-map.

\section{Acknowledgments}

We thank Daniel Butter, Michael Duff, Marc Grisaru, Finn Larsen and Ashoke Sen for valuable discussions. N.B. acknowledges the hospitality of the Indian Institute of Science (Bangalore) and the Tata Institute of Fundamental Research (Mumbai) extended to her during the completion of this work. N.B. is supported by a Veni grant of the 'Nederlandse Organisatie voor Wetenschappelijk Onderzoek (NWO)'. B.d.W. is supported by the ERC Advanced Grant no. 246974, "Supersymmetry: a window to non-perturbative physics". The work of S.K. is supported by the French ANR contract 05-BLAN-NT09-573739, the ERC Advanced Grant no. 226371, the ITN programme PITN-GA-2009-237920 and the IFCPAR programme 4104-2. Until October 12011 it was part of the research programme of the 'Stichting voor Fundamenteel Onderzoek der Materie (FOM)', which is financially supported by the 'Nederlandse Organisatie voor Wetenschappelijk Onderzoek (NWO)'.

\section{A Relations between 5D and 4D Riemann curvatures}

Based on (2.6) one can evaluate the relation between $5 D$ and $4 D$ curvature components. In the equations below, derivatives $\mathcal{D}_{a}$ are covariant with respect to $4 D$ local Lorentz transformations and dilatations. The results are as follows (in this appendix the $5 D$ curvature components are consistently denoted by $\hat{R}$ ),

$$
\begin{aligned}
\hat{R}_{\mu \nu}{ }^{a b}= & R_{\mu \nu}{ }^{a b}+\frac{1}{2} \phi^{-2}\left[F(B)_{\mu}{ }^{[a} F(B)_{\nu}{ }^{b]}+F(B)_{\mu \nu} F(B)^{a b}\right] \\
& -B_{[\mu}\left[2 \phi^{-3} F(B)_{\nu]}{ }^{[a} \mathcal{D}^{b]} \phi+\mathcal{D}_{\nu]}\left[\phi^{-2} F(B)^{a b}\right]\right]
\end{aligned}
$$




$$
\begin{aligned}
\hat{R}_{\mu \nu}{ }^{a 5}= & -\mathcal{D}_{[\mu}\left[\phi^{-1} F(B)_{\nu]}^{a}\right]-\phi^{-2} \mathcal{D}^{a} \phi F(B)_{\mu \nu} \\
& +B_{[\mu}\left[2 \mathcal{D}_{\nu]}\left[\phi^{-2} \mathcal{D}^{a} \phi\right]+\frac{1}{2} \phi^{-3} F(B)_{\nu] b} F(B)^{a b}\right], \\
\hat{R}_{\mu \hat{5}}{ }^{a b}= & \frac{1}{2} \mathcal{D}_{\mu}\left[\phi^{-2} F(B)^{a b}\right]+\phi^{-3} F(B)_{\mu}{ }^{[a} \mathcal{D}^{b]} \phi, \\
\hat{R}_{\mu \hat{5}}{ }^{a 5}= & -\mathcal{D}_{\mu}\left[\phi^{-2} \mathcal{D}^{a} \phi\right]-\frac{1}{4} \phi^{-3} F(B)_{\mu b} F(B)^{a b} .
\end{aligned}
$$

With tangent-space indices, $\hat{R}_{C D}{ }^{A B}$ takes the form,

$$
\begin{aligned}
\hat{R}_{c d}{ }^{a b} & =R_{c d}{ }^{a b}+\frac{1}{2} \phi^{-2}\left[F(B)_{c}{ }^{[a} F(B)_{d}{ }^{b]}+F(B)_{c d} F(B)^{a b}\right], \\
\hat{R}_{c d}{ }^{a 5} & =\frac{1}{2} \phi^{-1} \mathcal{D}^{a} F(B)_{c d}-\phi^{-2}\left[\mathcal{D}^{a} \phi F(B)_{c d}-F(B)^{a}{ }_{[c} \mathcal{D}_{d]} \phi\right], \\
\hat{R}_{c 5}{ }^{a b} & =\frac{1}{2} \phi^{-1} \mathcal{D}_{c} F(B)^{a b}-\phi^{-2}\left[F(B)^{a b} \mathcal{D}_{c} \phi-F(B)_{c}{ }^{[a} \mathcal{D}^{b]} \phi\right], \\
\hat{R}_{c 5}{ }^{a 5} & =-\phi D_{c}(\omega)\left[\phi^{-2} \mathcal{D}^{a} \phi\right]-\frac{1}{4} \phi^{-2} F(B)_{c b} F(B)^{a b} .
\end{aligned}
$$

Note that these components satisfy the pair-exchange property of the Riemann tensor. Contracted versions of the Riemann tensor take the form,

$$
\begin{aligned}
\hat{R}_{c B}{ }^{a B} & =R_{c b}{ }^{a b}+\frac{1}{2} \phi^{-2} F(B)_{c b} F(B)^{a b}-\phi \mathcal{D}_{c}\left[\phi^{-2} \mathcal{D}^{a} \phi\right], \\
\hat{R}_{A 5}{ }^{A b} & =\frac{1}{2} \phi^{-1} \mathcal{D}_{a} F(B)^{a b}-\frac{3}{2} \phi^{-2} F(B)^{a b} \mathcal{D}_{a} \phi, \\
\hat{R}_{A 5}{ }^{A 5} & =-\phi \mathcal{D}_{a}(\omega)\left[\phi^{-2} \mathcal{D}^{a} \phi\right]-\frac{1}{4} \phi^{-2} F(B)_{a b} F(B)^{a b}, \\
\hat{R}_{A B}{ }^{A B} & =R_{a b}{ }^{a b}-2 \phi \mathcal{D}_{a}\left[\phi^{-2} \mathcal{D}^{a} \phi\right]+\frac{1}{4} \phi^{-2} F(B)_{a b} F(B)^{a b} .
\end{aligned}
$$

Furthermore one may consider components of $\hat{R}_{[A B}{ }^{E F} \hat{R}_{C D] E F}$, which are required for the dimensional reduction of the $5 D$ mixed Chern-Simons term,

$$
\begin{aligned}
\hat{R}_{[a b}{ }^{E F} \hat{R}_{c d] E F}= & R_{[a b}{ }^{e f} R_{c d] e f}+\phi^{-2} R_{a b}^{e f}\left[F(B)_{c e} F(B)_{d f}+F(B)_{c d} F(B)_{e f}\right] \\
& +\frac{1}{4} \phi^{-4} F(B)_{a b}\left[2 F(B)_{c e} F(B)_{d f} F(B)^{e f}+F(B)_{c d} F(B)^{2}\right] \\
& +\frac{1}{2} \phi^{-2} \mathcal{D}_{e} F(B)_{a b} \mathcal{D}^{e} F(B)_{c d} \\
& +2 \phi^{-1} \mathcal{D}^{e} F(B)_{a b}\left[F(B)_{c e} \mathcal{D}_{d} \phi^{-1}+F(B)_{c d} \mathcal{D}_{e} \phi^{-1}\right] \\
& +\left.2 F_{a b}(B)\left[F_{c d}(B)\left(\mathcal{D}_{e} \phi^{-1}\right)^{2}+2 F(B)_{c e} \mathcal{D}^{e} \phi^{-1} \mathcal{D}_{d} \phi^{-1}\right]\right|_{[a b c d]}, \\
\hat{R}_{\hat{5}[a}^{E F} \hat{R}_{c d] E F}= & -\phi \mathcal{D}_{[a}\left[\frac{1}{2} \phi^{-2} R_{c d]}^{e f} F(B)_{e f}\right. \\
& +\frac{1}{8} \phi^{-4}\left[F(B)^{2} F(B)_{c d]}+2 F(B)^{e f} F(B)_{c e} F(B)_{d f}\right] \\
& \left.-2 \phi^{-1} F(B)_{c}^{e} \mathcal{D}_{d]}\left(\mathcal{D}_{e} \phi^{-1}\right)+F(B)_{c d]}\left(\mathcal{D} \phi^{-1}\right)^{2}\right] .
\end{aligned}
$$

where we made use of the Bianchi identity on $F(B)$ on the $4 D$ Riemann tensor. 


\section{B Conversion of $5 D$ symplectic Majorana spinors into $4 D$ chiral spinors}

In this paper we have to convert $5 D$ symplectic Majorana spinors into $4 D$ chiral spinors, so as to obtain the dimensionally reduced supersymmetry transformations in $4 D$ notation. We explain this here using Pauli-Källén notation where all gamma matrices are hermitian. As it turns out, one can use the same $4 \times 4$ charge-conjugation matrix $C$ in four and in five dimensions, satisfying

$$
C^{\mathrm{T}}=-C, \quad C^{\dagger}=C^{-1} .
$$

The $4 D$ gamma matrices are subject to,

$$
C \gamma_{a} C^{-1}=-\gamma_{a}^{\mathrm{T}}, \quad \gamma_{5}=\frac{1}{24} \varepsilon^{a b c d} \gamma_{a} \gamma_{b} \gamma_{c} \gamma_{d}
$$

where the indices $a, b, \ldots$ take four values. As is obvious, $\gamma_{5}$ satisfies $C \gamma_{5} C^{-1}=\gamma_{5}{ }^{\mathrm{T}}$. Majorana spinors $\psi$ have chiral components satisfying,

$$
C^{-1}\left(\bar{\psi}_{\mp}\right)^{\mathrm{T}}=\psi_{ \pm} .
$$

where $\psi_{ \pm}$are eigenspinors of $\gamma_{5}$ with eigenvalue \pm 1 . Since we will always be dealing with R-symmetry doublets of spinors labeled by an index $i, j, \ldots=1,2$, one uses the convention that the position of the index denotes at the same time the chirality. For reasons of convenience this is not done in a uniform way, so that the relation between the chirality and the position of the index differs from spinor to spinor.

In five dimensions, the charge conjugation properties of the gamma matrices $\hat{\gamma}_{A}$ are,

$$
C \hat{\gamma}_{A} C^{-1}=\hat{\gamma}_{A}{ }^{\mathrm{T}}, \quad \hat{\gamma}_{A B C D E}=\mathbf{1} \varepsilon_{A B C D E},
$$

where the indices $A, B, \ldots$ take five values. Note that the last equation defines $\gamma_{5}$ as a product of the remaining four gamma matrices. However, these gamma matrices are not identical to the $4 D$ ones, in view of the sign difference between the first equations of (B.2) and (B.4). Nevertheless, as we shall see below, the matrix $\gamma_{5}$ will remain the same as the $4 D$ one, defined in the second equation of (B.2).

It is easy to construct the remaining $5 D$ gamma matrices from the $4 D$ ones. Namely, one may assume that,

$$
\hat{\gamma}_{a}=\mathrm{i} \gamma_{a} \gamma_{5},
$$

since the matrices on the right-hand side are symmetric with respect to charge conjugation, they anti-commute with $\gamma_{5}$, and the product of two gamma matrices satisfies, $\hat{\gamma}_{a} \hat{\gamma}_{b}=\gamma_{a} \gamma_{b}$. Because the gamma matrices are different, also the definition of the Dirac conjugate will differ for $4 D$ and $5 D$ spinors, according to the relation,

$$
\left.\bar{\psi}\right|_{D=5}=\left.\bar{\psi}\right|_{D=4} \mathrm{i} \gamma_{5} .
$$

This fact will be relevant for the action when reducing to four dimensions, but also when relating the $5 D$ symplectic Majorana condition to the $4 D$ Majorana condition. 
The $5 D$ symplectic Majorana condition for spinors $\psi^{i}$ read,

$$
C^{-1}\left(\bar{\psi}_{i}\right)^{\mathrm{T}}=\varepsilon_{i j} \psi^{j}
$$

Upon replacing the $5 D$ Dirac conjugate to the $4 D$ one, one obtains,

$$
C^{-1}\left(\bar{\psi}_{i \mp}\right)^{\mathrm{T}}=\mp \mathrm{i} \varepsilon_{i j} \psi_{ \pm}^{j},
$$

where we have adopted chiral spinor components. Now let us assume that the $4 D$ spinor with upper index $i$ is of positive chirality, so that we identify it with the $5 D$ field $\psi_{+}^{i}$. In the $4 D$ context we know from (B.3), that the Dirac conjugate is then equal to the corresponding field of negative chirality, which we write with a lower $\mathrm{SU}(2)$ index. In this way we derive from (B.8) that the $5 D$ field can be decomposed in $4 D$ chiral spinors according to

$$
\left.\psi^{i}\right|_{5 D}=\psi_{+}^{i}+\mathrm{i} \varepsilon^{i j} \psi_{j-}
$$

where $\psi^{i}$ and $\psi_{i}$ appearing on the right-hand side are the positive- and negative-chirality components of a $4 D$ Majorana spinor doublet.

In case the four-dimensional negative-chirality spinor carries an upper $\mathrm{SU}(2)$ index, then the above relation changes into,

$$
\left.\psi^{i}\right|_{5 D}=\psi_{-}^{i}-\mathrm{i} \varepsilon^{i j} \psi_{j+}
$$

After this conversion defined by (B.9) and (B.10) it remains possible to redefine the $4 D$ Majorana spinors by a chiral $\mathrm{U}(1)$ and a scale transformation without affecting the Majorana condition.

\section{Supersymmetry transformations in four dimension}

In four space-time dimensions we follow the notation used e.g. in [8, 9]. Space-time and Lorentz indices are denoted by $\mu, \nu, \ldots$, and $a, b, \ldots$, respectively; $\mathrm{SU}(2)$-indices are denoted by $i, j, \ldots$. Furthermore, (anti-)symmetrizations are always defined with unit strength.

For the convenience of the reader we summarize the $4 D$ transformation rules of the superconformal fields and their relation to the superconformal algebra, as well as their covariant quantities contained in the so-called Weyl supermultiplet. The superconformal algebra comprises the generators of the general-coordinate, local Lorentz, dilatation, special conformal, chiral $\mathrm{SU}(2)$ and $\mathrm{U}(1)$, supersymmetry (Q) and special supersymmetry (S) transformations. The gauge fields associated with general-coordinate transformations $\left(e_{\mu}{ }^{a}\right)$, dilatations $\left(b_{\mu}\right)$, chiral symmetry $\left(\mathcal{V}_{\mu}{ }^{i}{ }_{j}\right.$ and $\left.A_{\mu}\right)$ and Q-supersymmetry $\left(\psi_{\mu}{ }^{i}\right)$ are independent fields. The remaining gauge fields associated with the Lorentz $\left(\omega_{\mu}^{a b}\right)$, special conformal $\left(f_{\mu}{ }^{a}\right)$ and S-supersymmetry transformations $\left(\phi_{\mu}{ }^{i}\right)$ are dependent fields. They are composite objects, which depend on the independent fields of the multiplet [31]. The 


\begin{tabular}{|c|cccccccc|ccc|cc|}
\hline & \multicolumn{10}{|c|}{ Weyl multiplet } & \multicolumn{2}{|c|}{ parameters } \\
\hline field & $e_{\mu}{ }^{a}$ & $\psi_{\mu}{ }^{i}$ & $b_{\mu}$ & $A_{\mu}$ & $\mathcal{V}_{\mu}{ }^{i} j$ & $T_{a b}{ }^{i j}$ & $\chi^{i}$ & $D$ & $\omega_{\mu}^{a b}$ & $f_{\mu}{ }^{a}$ & $\phi_{\mu}{ }^{i}$ & $\epsilon^{i}$ & $\eta^{i}$ \\
\hline$w$ & -1 & $-\frac{1}{2}$ & 0 & 0 & 0 & 1 & $\frac{3}{2}$ & 2 & 0 & 1 & $\frac{1}{2}$ & $-\frac{1}{2}$ & $\frac{1}{2}$ \\
\hline$c$ & 0 & $-\frac{1}{2}$ & 0 & 0 & 0 & -1 & $-\frac{1}{2}$ & 0 & 0 & 0 & $-\frac{1}{2}$ & $-\frac{1}{2}$ & $-\frac{1}{2}$ \\
\hline$\gamma_{5}$ & & + & 1 & & & + & & & & - & + & - \\
\hline
\end{tabular}

Table 4. Weyl and chiral weights $(w$ and $c)$ and fermion chirality $\left(\gamma_{5}\right)$ of the Weyl multiplet component fields and the supersymmetry transformation parameters.

corresponding supercovariant curvatures and covariant fields are contained in a tensor chiral multiplet, which comprises $24+24$ off-shell degrees of freedom. In addition to the independent superconformal gauge fields, it contains three other fields: a Majorana spinor doublet $\chi^{i}$, a scalar $D$, and a selfdual Lorentz tensor $T_{a b i j}$, which is anti-symmetric in $[a b]$ and $[i j]$. The Weyl and chiral weights have been collected in table 4 .

Under Q-supersymmetry, S-supersymmetry and special conformal transformations the independent fields of the Weyl multiplet transform as follows,

$$
\begin{aligned}
\delta e_{\mu}{ }^{a} & =\bar{\epsilon}^{i} \gamma^{a} \psi_{\mu i}+\bar{\epsilon}_{i} \gamma^{a} \psi_{\mu}{ }^{i} \\
\delta \psi_{\mu}{ }^{i} & =2 \mathcal{D}_{\mu} \epsilon^{i}-\frac{1}{8} T_{a b}{ }^{i j} \gamma^{a b} \gamma_{\mu} \epsilon_{j}-\gamma_{\mu} \eta^{i} \\
\delta b_{\mu} & =\frac{1}{2} \bar{\epsilon}^{i} \phi_{\mu i}-\frac{3}{4} \bar{\epsilon}^{i} \gamma_{\mu} \chi_{i}-\frac{1}{2} \bar{\eta}^{i} \psi_{\mu i}+\text { h.c. }+\Lambda_{K}^{a} e_{\mu a}, \\
\delta A_{\mu} & =\frac{1}{2} \mathrm{i} \bar{\epsilon}^{i} \phi_{\mu i}+\frac{3}{4} \mathrm{i} \bar{\epsilon}^{i} \gamma_{\mu} \chi_{i}+\frac{1}{2} \mathrm{i} \bar{\eta}^{i} \psi_{\mu i}+\text { h.c. } \\
\delta \mathcal{V}_{\mu}{ }^{i}{ }_{j} & =2 \bar{\epsilon}_{j} \phi_{\mu}{ }^{i}-3 \bar{\epsilon}_{j} \gamma_{\mu} \chi^{i}+2 \bar{\eta}_{j} \psi_{\mu}{ }^{i}-\text { (h.c. ; traceless), } \\
\delta T_{a b}{ }^{i j} & =8 \bar{\epsilon}^{[i} R(Q)_{a b}{ }^{j]}, \\
\delta \chi^{i} & =-\frac{1}{12} \gamma^{a b} \not D T_{a b}{ }^{i j} \epsilon_{j}+\frac{1}{6} R(\mathcal{V})_{\mu \nu}{ }_{j} \gamma^{\mu \nu} \epsilon^{j}-\frac{1}{3} \mathrm{i} R_{\mu \nu}(A) \gamma^{\mu \nu} \epsilon^{i}+D \epsilon^{i}+\frac{1}{12} \gamma_{a b} T^{a b i j} \eta_{j}, \\
\delta D & =\bar{\epsilon}^{i} \not D \chi_{i}+\bar{\epsilon}_{i} \not D \chi^{i} .
\end{aligned}
$$

Here $\epsilon^{i}$ and $\epsilon_{i}$ denote the spinorial parameters of Q-supersymmetry, $\eta^{i}$ and $\eta_{i}$ those of Ssupersymmetry, and $\Lambda_{K}{ }^{a}$ is the transformation parameter for special conformal boosts. The full superconformally covariant derivative is denoted by $D_{\mu}$, while $\mathcal{D}_{\mu}$ denotes a covariant derivative with respect to Lorentz, dilatation, chiral U(1), and $\mathrm{SU}(2)$ transformations,

$$
\mathcal{D}_{\mu} \epsilon^{i}=\left(\partial_{\mu}-\frac{1}{4} \omega_{\mu}^{c d} \gamma_{c d}+\frac{1}{2} b_{\mu}+\frac{1}{2} \mathrm{i} A_{\mu}\right) \epsilon^{i}+\frac{1}{2} \mathcal{V}_{\mu}{ }^{i} \epsilon^{j}
$$

Just as in five dimensions the gauge fields associated with local Lorentz transformations, S-supersymmetry and special conformal boosts, $\omega_{\mu}{ }^{a b}, \phi_{\mu}{ }^{i}$ and $f_{\mu}{ }^{a}$, respectively, are composite and determined by conventional constraints. In this case these constraints are 
S-supersymmetry invariant and they take the following form,

$$
\begin{aligned}
R(P)_{\mu \nu}{ }^{a} & =0, \\
\gamma^{\mu} R(Q)_{\mu \nu}{ }^{i}+\frac{3}{2} \gamma_{\nu} \chi^{i} & =0, \\
e^{\nu}{ }_{b} R(M)_{\mu \nu a}{ }^{b}-\mathrm{i} \tilde{R}(A)_{\mu a}+\frac{1}{8} T_{a b i j} T_{\mu}{ }^{b i j}-\frac{3}{2} D e_{\mu a} & =0 .
\end{aligned}
$$

The curvatures appearing in (C.3) take the following form,

$$
\begin{aligned}
R(P)_{\mu \nu}{ }^{a}= & 2 \partial_{[\mu} e_{\nu]}^{a}+2 b_{[\mu} e_{\nu]}^{a}-2 \omega_{[\mu}^{a b} e_{\nu] b}-\frac{1}{2}\left(\bar{\psi}_{[\mu}{ }^{i} \gamma^{a} \psi_{\nu] i}+\text { h.c. }\right), \\
R(Q)_{\mu \nu}{ }^{i}= & 2 \mathcal{D}_{[\mu} \psi_{\nu]}^{i}-\gamma_{[\mu} \phi_{\nu]}{ }^{i}-\frac{1}{8} T^{a b i j} \gamma_{a b} \gamma_{[\mu} \psi_{\nu] j} \\
R(M)_{\mu \nu}{ }^{a b}= & 2 \partial_{[\mu} \omega_{\nu]}^{a b}-2 \omega_{[\mu}^{a c} \omega_{\nu] c}^{b}-4 f_{[\mu}{ }^{a} e_{\nu]}^{b]}+\frac{1}{2}\left(\bar{\psi}_{[\mu}{ }^{i} \gamma^{a b} \phi_{\nu] i}+\text { h.c. }\right) \\
& +\left(\frac{1}{4} \bar{\psi}_{\mu}{ }^{i} \psi_{\nu}{ }^{j} T^{a b}{ }_{i j}-\frac{3}{4} \bar{\psi}_{[\mu}{ }^{i} \gamma_{\nu]} \gamma^{a b} \chi_{i}-\bar{\psi}_{[\mu}{ }^{i} \gamma_{\nu]} R(Q)^{a b}{ }_{i}+\text { h.c. }\right) .
\end{aligned}
$$

Chiral multiplets can be consistently reduced by imposing a reality constraint, which requires specific values for the Weyl and chiral weights. The two cases that are relevant are the vector multiplet, which arises upon reduction from a scalar chiral multiplet, and the Weyl multiplet, which is a reduced anti-selfdual chiral tensor multiplet. Both reduced multiplets require weight $w=1$.

The vector multiplet contains a complex scalar $X$, a chiral spinor $\Omega_{i}$ and a gauge field $W_{\mu}$, which transform under Q- and S-supersymmetry transformations as follows,

$$
\begin{aligned}
\delta X & =\bar{\epsilon}^{i} \Omega_{i} \\
\delta \Omega_{i} & =2 \not D X \epsilon_{i}+\frac{1}{2} \varepsilon_{i j} F_{\mu \nu}^{-} \gamma^{\mu \nu} \epsilon^{j}+Y_{i j} \epsilon^{j}+2 X \eta_{i}, \\
\delta W_{\mu} & =\varepsilon^{i j} \bar{\epsilon}_{i}\left(\gamma_{\mu} \Omega_{j}+2 \psi_{\mu j} X\right)+\varepsilon_{i j} \bar{\epsilon}^{i}\left(\gamma_{\mu} \Omega^{j}+2 \psi_{\mu}^{j} \bar{X}\right), \\
\delta Y_{i j} & =2 \bar{\epsilon}_{(i} \not D \Omega_{j)}+2 \varepsilon_{i k} \varepsilon_{j l} \bar{\epsilon}^{(k} \not D \Omega^{l)},
\end{aligned}
$$

Here $F_{\mu \nu}^{-}$denotes the anti-selfdual component associated with the field strength of $W_{\mu}$,

$$
\begin{aligned}
F_{a b}^{-}= & \left(\delta_{a b}^{c d}-\frac{1}{2} \varepsilon_{a b}{ }^{c d}\right) e_{c}{ }^{\mu} e_{d}^{\nu} \partial_{[\mu} W_{\nu]} \\
& +\frac{1}{4}\left[\bar{\psi}_{\rho}^{i} \gamma_{a b} \gamma^{\rho} \Omega^{j}+\bar{X} \bar{\psi}_{\rho}^{i} \gamma^{\rho \sigma} \gamma_{a b} \psi_{\sigma}^{j}-\bar{X} T_{a b}{ }^{i j}\right] \varepsilon_{i j},
\end{aligned}
$$

The Weyl multiplet was already discussed at the beginning of this appendix. The Weyl and chiral weights for the vector multiplet, the hypermultiplet and the Weyl multiplet in four dimensions has been summarized in tables 3 and 4 .

Open Access. This article is distributed under the terms of the Creative Commons Attribution License which permits any use, distribution and reproduction in any medium, provided the original author(s) and source are credited. 


\section{References}

[1] B. de Wit, F. Vanderseypen and A. Van Proeyen, Symmetry structure of special geometries, Nucl. Phys. B 400 (1993) 463 [hep-th/9210068] [INSPIRE].

[2] D. Gaiotto, A. Strominger and X. Yin, New connections between $4 D$ and $5 D$ black holes, JHEP 02 (2006) 024 [hep-th/0503217] [INSPIRE].

[3] D. Gaiotto, A. Strominger and X. Yin, 5D black rings and $4 D$ black holes, JHEP 02 (2006) 023 [hep-th/0504126] [INSPIRE].

[4] K. Behrndt, G. Lopes Cardoso and S. Mahapatra, Exploring the relation between $4 D$ and $5 D$ BPS solutions, Nucl. Phys. B 732 (2006) 200 [hep-th/0506251] [INSPIRE].

[5] T. Kugo and K. Ohashi, Supergravity tensor calculus in $5 D$ from $6 D$, Prog. Theor. Phys. 104 (2000) 835 [hep-ph/0006231] [INSPIRE].

[6] K. Hanaki, K. Ohashi and Y. Tachikawa, Supersymmetric completion of an $R^{2}$ term in five-dimensional supergravity, Prog. Theor. Phys. 117 (2007) 533 [hep-th/0611329] [INSPIRE].

[7] E. Bergshoeff, M. de Roo and B. de Wit, Extended conformal supergravity, Nucl. Phys. B 182 (1981) 173 [InSPIRE].

[8] G. Lopes Cardoso, B. de Wit, J. Käppeli and T. Mohaupt, Stationary BPS solutions in $N=2$ supergravity with $R^{2}$ interactions, JHEP 12 (2000) 019 [hep-th/0009234] [INSPIRE].

[9] B. de Wit, S. Katmadas and M. van Zalk, New supersymmetric higher-derivative couplings: full $N=2$ superspace does not count!, JHEP 01 (2011) 007 [arXiv:1010.2150] [INSPIRE].

[10] A. Castro, J.L. Davis, P. Kraus and F. Larsen, 5D black holes and strings with higher derivatives, JHEP 06 (2007) 007 [hep-th/0703087] [INSPIRE].

[11] A. Castro, J.L. Davis, P. Kraus and F. Larsen, Precision entropy of spinning black holes, JHEP 09 (2007) 003 [arXiv:0705.1847] [INSPIRE].

[12] A. Castro, J.L. Davis, P. Kraus and F. Larsen, String theory effects on five-dimensional black hole physics, Int. J. Mod. Phys. A 23 (2008) 613 [arXiv:0801.1863] [InSPIRE].

[13] B. de Wit and S. Katmadas, Near-horizon analysis of $D=5$ BPS black holes and rings, JHEP 02 (2010) 056 [arXiv:0910.4907] [INSPIRE].

[14] B. de Wit, B. Kleijn and S. Vandoren, Superconformal hypermultiplets, Nucl. Phys. B 568 (2000) 475 [hep-th/9909228] [InSPIRE].

[15] B. de Wit, P. Lauwers and A. Van Proeyen, Lagrangians of $N=2$ supergravity-matter systems, Nucl. Phys. B 255 (1985) 569 [INSPIRE].

[16] B. de Wit and A. Van Proeyen, Potentials and symmetries of general gauged $N=2$ supergravity: Yang-Mills models, Nucl. Phys. B 245 (1984) 89 [InSPIRE].

[17] S. Ferrara and B. Zumino, Structure of conformal supergravity, Nucl. Phys. B 134 (1978) 301 [inSPIRE].

[18] S. Ferrara and P. Van Nieuwenhuizen, Structure of supergravity, Phys. Lett. B 78 (1978) 573 [INSPIRE] 
[19] P. Townsend and P. van Nieuwenhuizen, Anomalies, topological invariants and the Gauss-Bonnet theorem in supergravity, Phys. Rev. D 19 (1979) 3592 [InSPIRE].

[20] R. Grimm, J. Wess and B. Zumino, A complete solution of the Bianchi identities in superspace, Nucl. Phys. B 152 (1979) 255 [INSPIRE].

[21] A. Sen, Logarithmic corrections to $N=2$ black hole entropy: an infrared window into the microstates, arXiv:1108.3842 [INSPIRE].

[22] J.P. Gauntlett and J.B. Gutowski, General concentric black rings, Phys. Rev. D 71 (2005) 045002 [hep-th/0408122] [INSPIRE].

[23] I. Bena and P. Kraus, Microscopic description of black rings in AdS/CFT, JHEP 12 (2004) 070 [hep-th/0408186] [INSPIRE].

[24] M. Cyrier, M. Guica, D. Mateos and A. Strominger, Microscopic entropy of the black ring, Phys. Rev. Lett. 94 (2005) 191601 [hep-th/0411187] [INSPIRE].

[25] I. Bena, P. Kraus and N.P. Warner, Black rings in Taub-NUT, Phys. Rev. D 72 (2005) 084019 [hep-th/0504142] [inSPIRE].

[26] I. Bena and P. Kraus, $R^{2}$ corrections to black ring entropy, hep-th/0506015 [INSPIRE].

[27] K. Hanaki, K. Ohashi and Y. Tachikawa, Comments on charges and near-horizon data of black rings, JHEP 12 (2007) 057 [arXiv:0704.1819] [INSPIRE].

[28] M. Henneaux and C. Teitelboim, Quantization of topological mass in the presence of a magnetic pole, Phys. Rev. Lett. 56 (1986) 689 [INSPIRE].

[29] X. Bekaert, Issues in electric magnetic duality, hep-th/0209169 [INSPIRE].

[30] X. Bekaert and A. Gomberoff, Quantization of the Chern-Simons coupling constant, JHEP 01 (2003) 054 [hep-th/0212099] [INSPIRE].

[31] B. de Wit, J. van Holten and A. Van Proeyen, Structure of $N=2$ supergravity, Nucl. Phys. B 184 (1981) 77 [Erratum ibid. B 222 (1983) 516] [INSPIRE]. 